\title{
APROVECHAMIENTO SOSTENIBLE Y CONSERVACIÓN DE PLANTAS medicinales en Cantarranas, Huehuetla, Hidalgo, MÉXICO, COMO UN MEDIO PARA MEJORAR LA CALIDAD DE VIDA EN LA COMUNIDAD
}

\author{
Berenice Nathaly López-Gutiérrez'1 Blanca Estela Pérez-Escandón y \\ Miguel Ángel Villavicencio Nieto \\ Laboratorio de Etnobotánica, Centro de Investigaciones Biológicas, \\ Universidad Autónoma del Estado de Hidalgo, Pachuca, Hidalgo, México \\ 'Autor para la correspondencia: Lgbn_18@hotmail.com
}

\begin{abstract}
Resumen: Este estudio se realizó en Cantarranas, Huehuetla, Hidalgo, México, donde el tipo de vegetación dominante es bosque tropical perennifolio. Cantarranas presenta alto grado de marginación, limitado acceso a servicios de salud y pocas oportunidades para incrementar sus ingresos económicos. El objetivo fue planear el aprovechamiento sostenible y conservación de plantas medicinales usadas en la comunidad como una alternativa para resolver problemas ambientales y sociales. Las especies fueron evaluadas con métodos etnobotánicos y ecológicos. Se usaron entrevistas estructuradas para documentar el valor cultural de las especies, nivel de fidelidad, prácticas e intensidad de manejo, usos y padecimientos tratados. La distribución y abundancia se obtuvo con el método de transectos lineales. Las 19 especies evaluadas son usadas para atender 51 padecimientos, los desórdenes gastrointestinales se tratan mayormente mediante las plantas medicinales. Las especies con mayor índice de valor cultural fueron Equisetum myriochaetum (0.69), Psidium guajava (0.37), Jaegeria macrocephala (0.26592) y Costus scaber (0.25496). Hamelia patens registró el mayor nivel de fidelidad (96\%), seguida de E. myriochaetum (88\%). Los valores de intensidad de manejo fueron más altos en C. scaber (9.2) y P. guajava (9). La densidad poblacional osciló entre 1 y 387 individuos por hectárea, según la especie y forma de vida. Mediante la vinculación sociedad y Universidad, se desarrolló e impartió un diplomado para los miembros de la comunidad, relacionado con el uso y aprovechamiento sostenible de plantas medicinales. Se constituyó una Sociedad Cooperativa con mujeres de la comunidad, ellas trabajan en la conservación y aprovechamiento sostenible de plantas medicinales.
\end{abstract}

Palabras clave: etnobotánica, remedios herbolarios, Otomí-Tepehua, valor cultural, vinculación.

\begin{abstract}
The present study was carried in Cantarranas, Huehuetla, Hidalgo, Mexico. The vegetation type is tropical evergreen forest. Cantarranas presents high degree of marginalization, limited access to health services, and few opportunities to increase its economic income. The aim was to design a strategy for the sustainable harvesting and conservation of medicinal plants used by the community, as an alternative to solve social and environmental problems. The species were evaluated by ethnobotanical and ecological methods. Using structured interviews, index of cultural value, index of Friedman value, practices and management intensity, and uses and conditions treated were documented. The distribution and abundance were obtained with line transects method. Nineteen plants were evaluated, they are used to treat 51 conditions; the gastrointestinal disorders were the most frequently treated with medicinal plants. The species with the higher index of cultural value were Equisetum myriochaetum (0.69), Psidium guajava (0.37), Jaegeria macrocephala (0.26) y Costus scaber (0.25). Hamelia patens recorded the highest level of fidelity (96\%), followed by E. myriochaetum (88\%). The values of management intensity are higher in species such as C. scaber (9.2) y P. guajava (9). The population density has a range between 1 and 387 individuals per hectare, depending on the species and life forms. Through linking society and University, we developed and offered a certified program for members of the community related to the use and sustainable harvesting of medicinal plants. A Cooperative Society was incorporated with women of the community and they work in the conservation and sustainable harvesting of medicinal plants in the community.
\end{abstract}

Key words: cultural value, ethnobotany, herbal remedies, linking, Otomí-Tepehua.

$\mathbf{L}$ a Sierra Otomí-Tepehua se localiza al este del estado de Hidalgo, México; con municipios como Agua Blanca, Tenango, San Bartolo Tutotepec y Huehuetla. Es una zona escarpada que forma parte de la Sierra Madre Oriental, aquí se encuentra la región más lluviosa de la entidad, con precipitación media anual que va de 2,000 a 3,500 mm, presenta 
bosque de coníferas y encinos, bosque mesófilo de montaña y bosque tropical perennifolio (INEGI, 1992). Si bien, a la fecha no se ha hecho un diagnóstico de la situación ambiental del área, es claro que la vegetación original se encuentra fragmentada, observándose pérdida de hábitat debido, principalmente, a las actividades ganaderas y agrícolas. En la zona existen comunidades vegetales interesantes, como el bosque mesófilo de montaña ubicado en Tenango, con la mayor riqueza de especies de plantas (453) comparado con otros doce bosques de México; actualmente este bosque se encuentra muy perturbado, afectado por incendios y la tala que se hace para utilizar los terrenos como potreros (Alcántara y Luna, 1997). En San Bartolo Tutotepec se localizan fragmentos de bosque de Fagus grandifolia subsp. mexica$n a$ con especies de plantas incluidas en la Norma Oficial Mexicana 059 (SEMARNAT, 2010), como Cyathea fulva, F. grandifolia, Magnolia schiedeana y Tillandsia imperialis, entre otras (Villavicencio y Pérez, 2005).

En esta zona se encuentran dos municipios con muy alto grado de marginación, San Bartolo Tutotepec y Huehuetla (COESPO, 2010). La región tiene una fuerte influencia indígena, pues registra ocupación de varios grupos mesoamericanos desde hace más de 1,000 años y en la actualidad, más de la mitad de las personas que la habitan son Otomíes de la Sierra o Tepehuas (INEGI, 2004). Huehuetla, por su grado de marginación, ocupa el tercer lugar entre los 84 municipios del estado de Hidalgo (COESPO, 2010), y es uno de los nueve municipios con mayores porcentajes de población indígena, donde el 74.2\% de los habitantes son Otomíes de la Sierra o Tepehuas (INEGI, 2004; Guzmán, 2009).

Los actuales habitantes de la región son herederos directos del patrimonio histórico-cultural de los antiguos pobladores del área; parte de ese legado es el conocimiento tradicional acerca del uso y manejo de las plantas, en especial las medicinales. Las plantas medicinales en comunidades rurales constituyen la base del cuidado primario de la salud, la recuperación del medio ambiente y el incremento en el ingreso familiar (WHO, 2002; Hamilton, 2004; van de Kop, 2006; Shengji et al., 2010). Por ejemplo, en San Antonio el Grande, Huehuetla, se utilizan 317 especies de plantas medicinales (Romero et al., 1999), y en las cabeceras municipales de San Bartolo Tutotepec y Huehuetla se hace uso medicinal de 160 (Callejas et al., 2008) y 170 (Zavala-Fragoso, 2011) especies de plantas, respectivamente. Reconocer y valorar la importancia que las plantas medicinales brindan a los pobladores en dichas comunidades, es un punto de partida para promover la conservación y el aprovechamiento sostenible de la biodiversidad. Es decir, la presencia, valoración y uso sostenible de las plantas medicinales puede ser la clave para conservar hábitats completos (Wiersum et al., 2006; LagosWitte et al., 2011; Payyappallimana y Fadeeva, 2013) y contribuir en la disminución del grado de pobreza y mejorar en la calidad de vida (Adams et al., 2004).

Tal como se ha considerado en algunos trabajos, una de las funciones más importantes de las plantas medicinales en la biología de la conservación es su uso y manejo tradicional, derivado de la importancia cultural que representan para las personas (Hamilton, 2004; Singh et al., 2012; Bhat et al., 2013; Blancas, et al., 2013). Por tanto, existe la necesidad de integrar programas de manejo sostenible y conservación de las plantas medicinales, basados en métodos que cuantifiquen su importancia cultural y económica, importancia ecológica, situación actual de sus poblaciones, distribución geográfica, estrategias de recolecta sostenible, propagación y cultivo (CMMAD, 1987; Ley General de Equilibrio Ecológico y la Protección al Ambiente, 1988; OMS et al., 1993; Caballero y Cortés, 2001; Schäfer, 2001; CBD, 2002; OMS, 2003; Bermúdez et al., 2005; Shengji et al., 2010; Raina et al., 2011; Gómez, 2012; Bhat et al., 2013). Por tanto, se inició el presente estudio en la Sierra Otomí-Tepehua en la comunidad de Cantarranas, Huehuetla. El objetivo fue planear y desarrollar el aprovechamiento sostenible y conservación de 19 plantas medicinales, con fundamento en investigación etnobotánica, ecológica y vinculación con la sociedad, para contribuir a solucionar la problemática ambiental y social presentes en la localidad.

\section{Materiales y Método}

Zona de estudio. Cantarranas se localiza al sureste del municipio de Huehuetla, en la zona ecológica tropical húmeda del estado de Hidalgo; entre $20^{\circ} 26^{\prime} 06^{\prime \prime}-20^{\circ} 26^{\prime} 43^{\prime \prime} \mathrm{N}$ y $98^{\circ} 03^{\prime} 34^{\prime \prime}-98^{\circ} 04^{\prime} 11^{\prime \prime} \mathrm{O}$, y distribución altitudinal de 390 a 1,260 m s.n.m. (Figura 1). El clima es cálido húmedo, temperatura promedio de $23.7^{\circ} \mathrm{C}$, precipitación media anual de $2,558 \mathrm{~mm}$, la vegetación predominante es bosque tropical perennifolio (Rzedowski, 1978; Pavón y Meza, 2009). Presenta un total de 335 habitantes, de los cuales 177 son hombres y 158 mujeres (INEGI, 2000); la población es mestiza con fuerte influencia indígena Otomí-Tepehua, reflejada en sus usos y costumbres. Es una comunidad rural con muy alto grado de marginación, no cuenta con ningún servicio médico y los pobladores deben salir a otras comunidades (San Bartolo Tutotepec o Huehuetla), para atender sus padecimientos o hacerlo mediante el uso de plantas medicinales. Entre las principales actividades de la comunidad se encuentra la agricultura y ganadería, que se realizan principalmente para el autoconsumo, por lo que existen muy pocas oportunidades para aumentar el ingreso económico familiar.

Etnobotánica. La metodología y sistematización de la información acerca del uso de plantas medicinales en comunidades rurales se ha encaminado en diferentes modalidades o proyectos de investigación etnobotánica, en donde los pobladores forman parte de sus propios procesos locales y confirman la importancia de la etnobotánica en el desarrollo social (Gispert et al., 1979, 2010; Martínez, 1990; Rodríguez y Torres, 1990; González-Chévez y Hersch-Martínez, 
1993; Hersch-Martínez y González, 1996; Monroy y Anaya, 2003). En este sentido, se seleccionaron 19 especies de plantas medicinales en torno a las que se realizó el presente trabajo etnobotánico. Para su elección, se consideró su importancia medicinal mencionada en trabajos previos, demanda a nivel local y regional, así como su distribución en la zona de estudio (Linares et al., 1990; Nicholson y Arzeni, 1993; Sánchez y Estrada, 1995; Mendoza et al., 1997; Romero et al., 1999; Pérez et al., 2003; Villavicencio y Pérez, 2005, 2006; Martínez-Moreno et al., 2006; Callejas et al., 2008; Linares y Bye, 2009; Mendoza y Lugo; 2011; Zavala-Fragoso, 2011). Se recolectaron las 19 especies con apoyo de los miembros de la comunidad. Las especies fueron identificadas por uno de los autores (Miguel Ángel Villavicencio Nieto) y los ejemplares de respaldo se encuentran depositados en el herbario del Centro de Investigaciones Biológicas de la Universidad Autónoma del Estado de Hidalgo, HGOM, (colección etnobotánica), con los números de colecta de los autores del presente trabajo. Para recabar la información etnobotánica, se aplicaron 26 entrevistas estructuradas a informantes clave (Alexiades, 1996), considerados los miembros de la comunidad con mayor conocimiento acerca del uso y manejo de las plantas medicinales. Los informantes clave fueron seleccionados por el método no probabilístico "bola de nieve" (Goodman, 1961), en donde un individuo de la población nomina a otro con la misma probabilidad de ser seleccionado, y así adquirir un grupo de estudio que se aproxime a una muestra aleatoria.

Índice de valor cultural. Se registró el promedio de mención acerca de características cualitativas que definen el valor cultural de las especies, los valores de las variables se basaron en trabajos previos (Pieroni, 2001; González-Insuasti y Caballero, 2006; Blancas et al., 2013). El índice de valor cultural (IC) de las especies se calculó mediante la modificación de la fórmula aplicada por Blancas et al. (2013). La modificación del índice fue:

$$
\mathrm{IC}=\mathrm{PUNuSNsuHtCM} / 10000
$$

donde $\mathrm{P}$ es el número de personas que usan las especie; $\mathrm{U}$ es la frecuencia de uso a través del año: $1=$ una vez al año, 2 = más de una vez al año, 3 = una vez al mes, $4=$ una vez por semana, 5 = más de una vez por semana; $\mathrm{Nu}$, el número de diferentes usos; $\mathrm{S}$, la estructura vegetal usada con mayor frecuencia: 1 = parte vegetativa (hoja/tallo), 2 $=$ reproductiva (flores/frutos/semillas), 3 = completa; Nsu, número de estructuras utilizadas; Ht, forma de reunión: $1=$ ocasionalmente (reunida mientras otras actividades se llevan a cabo), 2 =xclusivamente (se realiza una salida especial para buscarla); C, posibilidad de comercio: $1=$ no existente, $2=$ si existente $\mathrm{y} ; \mathrm{M}$, uso medicinal: $1=$ no existente, $2=$ si existente. De igual manera, se registró el promedio de la abundancia de las especies percibida por los informantes: 1 $=$ rara, $2=$ escasa, $3=$ abundante y $4=$ muy abundante.
Nivel de fidelidad de las especies. Se calculó el valor de Friedman (FL) o nivel de fidelidad de las plantas medicinales, para determinar aquellas potencialmente más efectivas. Éste sugiere que una especie ha sido sometida a una selección a través del tiempo; y por tanto, a mayor valor obtenido, la probabilidad de que sea efectiva es mayor (Friedman et al., 1986). Se calculó con la fórmula:

$$
\mathrm{FL}=(\mathrm{Ip} / \mathrm{It}) \times 100
$$

donde Ip es el número de informantes que mencionaron una especie con uso medicinal (frecuencia de mención) e It el número total de informantes.

Proporción de acuerdo sobre los aparatos o sistemas tratados por plantas medicinales. Se analizó la homogeneidad del conocimiento de los informantes con respecto a las plantas medicinales potencialmente efectivas, utilizadas para tratar determinados aparatos o sistemas. El resultado se registró entre 0 y 1 , en donde el valor más alto indicó mayor acuerdo (Heinrich et al., 1998; Kumar, 2011). Se obtuvo con base en el método modificado propuesto por Boster (1985), en donde los pares (209) fueron considerados entre las plantas medicinales (19), y el aparato o sistema tratado (11) con respecto a las menciones dadas por los informantes:

$$
\mathrm{PA}_{1}=\mathrm{a}_{1} / \mathrm{n}_{\mathrm{s}}
$$

donde $\mathrm{PA}_{1}$ es la proporción de acuerdo para el par $n$ (planta y aparato o sistema), $\mathrm{a}_{1}$ es el número de menciones en que la planta $\mathrm{n}$ trata el aparato o sistema $n$, y $\mathrm{n}_{\mathrm{s}}$ es el total de citas de todos los aparatos o sistemas tratados por la planta $n$.

Prácticas e intensidad manejo. Las prácticas de manejo a las cuales son sometidas las plantas se consideran acciones

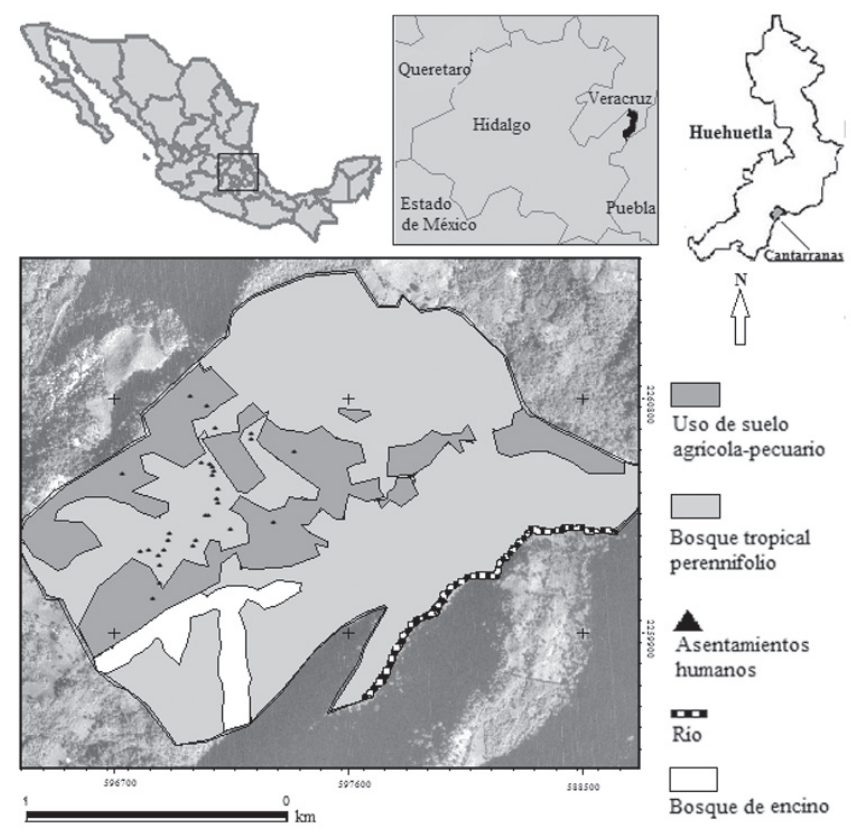

Figura 1. Ubicación y caracterización de Cantarranas, Huehuetla, estado de Hidalgo. 
directas o indirectas realizadas por los humanos para aumentar su disponibilidad; se llevan a cabo in situ en ambientes silvestres y ruderales, así como ex situ en las zonas antropogénicas (González-Insuasti y Caballero, 2006). Dichas prácticas pueden ser selectivas cuando individuos de plantas con fenotipos característicos se favorecen mediante la manipulación y no selectivas cuando los individuos son manejados de forma oportunista. La recolección simple en poblaciones silvestres y la agricultura son prácticas de manejo separadas; por lo que se consideró el siguiente gradiente de manejo entre ambas: 1 = recolección (en poblaciones silvestres), 2 = manejo no selectivo incipiente, $3=$ manejo selectivo incipiente, $4=$ cultivo y $5=$ agricultura (Bye, 1998; Caballero y Cortés, 2001; González-Insuasti y Caballero, 2006). La intensidad de manejo, indica el grado en que una especie vegetal es manipulada y depende de dos factores importantes: las prácticas de manejo usadas y el número de personas que usan algunos de estos sistemas (González-Insuasti y Caballero, 2006; Blancas et al., 2013). La intensidad de manejo se calculó para cada una de las plantas mediante la fórmula propuesta por González-Insuasti y Caballero (2006):

$$
\mathrm{IM}=\Sigma \mathrm{MF}(\mathrm{n} / \mathrm{N})
$$

en donde IM es la intensidad de manejo para una planta particular; MF es la práctica de manejo por grado de complejidad: 1 = recolección en poblaciones silvestres, $2=$ manejo no selectivo incipiente, $3=$ manejo selectivo incipiente, $4=$ cultivo, 5 = agricultura; $\mathrm{n}$ es el número de informantes que prefieren un manejo y; $\mathrm{N}$ el número total de informantes.

Distribución geográfica, cobertura y densidad poblacional. Con el fin de hacer una breve evaluación de algunos parámetros poblacionales en la zona de estudio y georeferenciar las poblaciones silvestres de las plantas medicinales estudiadas, se recorrieron durante los meses de enero a septiembre de 2012, $7 \mathrm{~km}$ derivados de ocho transectos variables (Sutherland, 1996; Krebs, 1999; Mostacedo y Fredericksen, 2000). A partir de la línea central del transecto, se marcó una distancia perpendicular, hacia cada lado, de $1 \mathrm{~m}$ para contabilizar hierbas, $2 \mathrm{~m}$ para arbustos y $3 \mathrm{~m}$ para árboles; estableciendo así el área para estimar la densidad, cobertura y distribución de las especies. La densidad poblacional se obtuvo por el conteo directo expresado como el número de individuos/unidad de área, el porcentaje de terreno cubierto mediante el método de estimación visual (DAFOR), en el cual el investigador tiene su propia interpretación de la cobertura de la población de acuerdo con los siguientes valores: $\mathrm{D}$ (dominante) $=\geq 80 \%$ cubierto por la población en el área establecida, $\mathrm{A}$ (abundante $)=50-80 \%, \mathrm{~F}$ (frecuente $)=$ $25-50 \%, \mathrm{O}($ ocasional $)=15-25 \%$ y R $($ rara $)=1-15 \%$.

Vinculación con la comunidad. La etnobotánica se considera un espacio favorable para la aplicación de elementos de investigación participativa, involucrando a los pobladores de las comunidades en términos de educación, capacitación e inserción de la ciencia (de Schutter, 1981; Hersch-Martínez y González, 1996; Durston y Miranda, 2002), desde los diferentes niveles de la participación social (Rubin de Celis, 1988) e implementando la vinculación como una función de la Institución de Educación Superior para la transferencia de avances científicos y tecnológicos, asesoría y consultoría (Campos y Sánchez, 2005, 2006). Se realizaron reuniones periódicas con autoridades y pobladores de Cantarranas durante el primer año de trabajo (2012), con el propósito de promover y reconocer la importancia del uso de las plantas medicinales en un contexto biológico y cultural. Durante el segundo año (2013) se llevaron a cabo acciones de aprovechamiento sostenible y conservación de plantas medicinales con la sociedad organizada, considerando sus conocimientos tradicionales y necesidades para contribuir a resolver la problemática social y ambiental presente en la comunidad.

Análisis de datos. El análisis estadístico de los datos se realizó con el programa MYSTAT 12. Mediante la prueba de Kolmogorov-Smirnov se determinó que las datos obtenidos no presentaron distribución normal, por lo cual fueron analizados con pruebas estadísticas no paramétricas (Zar, 1996). Se realizaron pruebas de Kruskal Wallis, considerando un nivel de significancia de 0.05 . Las variables que presentaron diferencias significativas fueron sometidas a una comparación múltiple de Tukey para establecer entre qué categorías se encontraban dichas diferencias. Con el fin de identificar características de las plantas medicinales que determinen la existencia de patrones relacionados con factores biológicos y culturales, se realizó un análisis de componentes principales utilizando el programa JPM 7 (SAS Institute, 2007). La matriz base se construyó colocando a las especies en las filas (19) y en las columnas el índice de valor cultural, intensidad de manejo y densidad poblacional. Mediante el uso de Sistemas de Información Geográfica (SIG) se elaboraron mapas de distribución de las especies en la zona de estudio, ya que proporcionan información fundamental para tomar decisiones acerca de su aprovechamiento y conservación. Los mapas se elaboraron utilizando el programa ARCVIEW GIS versión 3.2 (ESRI, 1999).

\section{Resultados}

El índice de valor cultural de las especies estudiadas osciló entre 0.01 y 0.69 (Cuadro 1). Los valores más altos se observaron en Equisetum myriochaetum, Psidium guajava, Jaegeria macrocephala y Costus scaber. Estas plantas se utilizan con mayor frecuencia (más de una vez al mes), se usa la planta completa o sus estructuras reproductivas y presentan posibilidad de comercio, a excepción de J. macrocephala. Los valores culturales más bajos se presentaron en especies usadas por pocos informantes, baja frecuencia de uso (una vez al año) y de las cuales se utilizan únicamente las hojas. Este es el caso de Cecropia obtusifolia, Kalanchoe pinnata 
Cuadro 1. Datos de las 19 especies de plantas medicinales estudiadas en Cantarranas, Huehuetla; derivados de las entrevistas estructuradas. IC: índice de valor cultural, FL: índice de Friedman o nivel de fidelidad (\%), FU: frecuencia de uso a través del año. $1=$ una vez al año, $2=$ más de una vez al año, 3 = una vez al mes. DP: disponibilidad percibida por los informantes. 2 = escasa, $3=$ abundante, $4=$ muy abundante. R: forma de reunión. 2 = exclusiva, EV: estructura vegetal más utilizada. 1 = Parte vegetativa (hoja/tallo), $2=$ reproductiva (flores/frutos/semillas), $3=$ completa. DP: categorías de uso de los informantes. $\mathrm{M}=$ medicinal, $\mathrm{Co}=$ comestible, $\mathrm{F}=$ forrajero, $\mathrm{Cb}=$ combustible, $\mathrm{Cm}=\mathrm{comercio}$.

\begin{tabular}{|c|c|c|c|c|c|c|c|c|}
\hline Nombre científico & Nombre común & Categorías de uso & IC & $\mathbf{F L}$ & FU & DP & $\mathbf{R}$ & EV \\
\hline Bidens pilosa & rosilla & $M, F$ & 0.2016 & 69 & 2 & 4 & 2 & 3 \\
\hline Cecropia obtusifolia & hormiguillo & $\mathrm{M}, \mathrm{Cb}, \mathrm{Cm}$ & 0.0208 & 27 & 1 & 3 & 2 & 1 \\
\hline Citrus limetta & lima limón & $\mathrm{Co}, \mathrm{M}$ & 0.1920 & 54 & 2 & 2 & 2 & 3 \\
\hline Citrus $\times$ aurantium & naranjo agrio & M & 0.0408 & 62 & 2 & 3 & 2 & 3 \\
\hline Costus scaber & caña de jabalí & $\mathrm{M}, \mathrm{Cm}$ & 0.2496 & 85 & 3 & 3 & 2 & 1 \\
\hline Cymbopogon citratus & zacate limón & M & 0.0528 & 15 & 3 & 3 & 2 & 3 \\
\hline Equisetum myriochaetum & cola de caballo & $\mathrm{M}, \mathrm{Cm}$ & 0.6912 & 88 & 3 & 2 & 2 & 3 \\
\hline Guazuma ulmifolia & guázima & $\mathrm{M}, \mathrm{Cb}$ & 0.1024 & 46 & 2 & 3 & 2 & 2 \\
\hline Hamelia patens & tres hojitas & M & 0.0300 & 96 & 3 & 3 & 2 & 2 \\
\hline Jaegeria macrocephala & árnica & M & 0.2592 & 73 & 3 & 3 & 2 & 3 \\
\hline Justicia spicigera & muitle & M & 0.2016 & 85 & 3 & 3 & 2 & 3 \\
\hline Kalanchoe pinnata & tronadora & M & 0.0160 & 35 & 2 & 4 & 2 & 1 \\
\hline Passiflora edulis & maracuyá & $\mathrm{Co}, \mathrm{M}$ & 0.1472 & 27 & 2 & 2 & 2 & 2 \\
\hline Persea americana & aguacate & $\mathrm{Co}, \mathrm{M}, \mathrm{Cm}, \mathrm{Cb}$ & 0.2112 & 54 & 2 & 3 & 2 & 2 \\
\hline Phyla dulcis & hierba dulce & M & 0.0368 & 58 & 2 & 3 & 2 & 1 \\
\hline Plantago major & Ilantén & M & 0.0112 & 42 & 2 & 3 & 2 & 1 \\
\hline Pseudogynoxys chenopodioides & árnica de bejuco & M & 0.1088 & 62 & 2 & 2 & 2 & 3 \\
\hline Psidium guajava & guayaba & $\mathrm{Co}, \mathrm{M}, \mathrm{Cm}, \mathrm{Cb}$ & 0.3744 & 85 & 3 & 3 & 2 & 1 \\
\hline Sambucus nigra subsp. canadensis & saúco & $\mathrm{Co}, \mathrm{M}$ & 0.1200 & 81 & 2 & 3 & 2 & 1 \\
\hline
\end{tabular}

y Plantago major. En el cuadro 1 se observan las plantas con mayor nivel de fidelidad (FL) y por tanto, las consideradas con mayor probabilidad de ser efectivas: Hamelia patens (96\%), E. myriochaetum (88\%), C. scaber, Justicia spicigera y P. guajava (85\%). Se observó una correlación positiva y significativa entre el índice de valor cultural y el nivel de fidelidad ( $\mathrm{R}=0.51, P=0.02$ ); por lo que las plantas medicinales con mayor valor cultural son consideradas potencialmente más efectivas. El 74\% de las especies son percibidas por los informantes como abundantes. Bidens pilosa y K. pinnata se consideran muy abundantes, y Citrus limetta, E. myriochaetum y Passiflora edulis como escasas (Cuadro 1). El 100\% de las especies se obtienen mediante actividades exclusivas, en las que se realizan salidas especialmente para buscarlas (Cuadro 1).

Se registraron cinco categorías de uso, de las cuales la medicinal presentó mayor número de plantas y difiriere significativamente del resto $(\mathrm{H}=52, P=<0.05$; Figura 2$)$. Los informantes mencionaron que las 19 plantas estudiadas son utilizadas para el tratamiento de 51 padecimientos. $\mathrm{Al}$ agrupar los padecimientos mencionados por los informantes en aparatos, sistemas y otros, se determinó que el aparato digestivo se trata con un mayor número de plantas medicinales $(\mathrm{H}=47.044, P=<0.001$; Figura 3$)$. De igual manera, el aparato digestivo agrupó el mayor número de padecimientos (13), tales como: dolor de estómago, gastritis, diarrea, disentería, ulceras gástricas, estreñimiento, parásitos intestinales, agruras, cólicos, indigestión, colitis, vómito y gases. Posteriormente, el aparato reproductor con

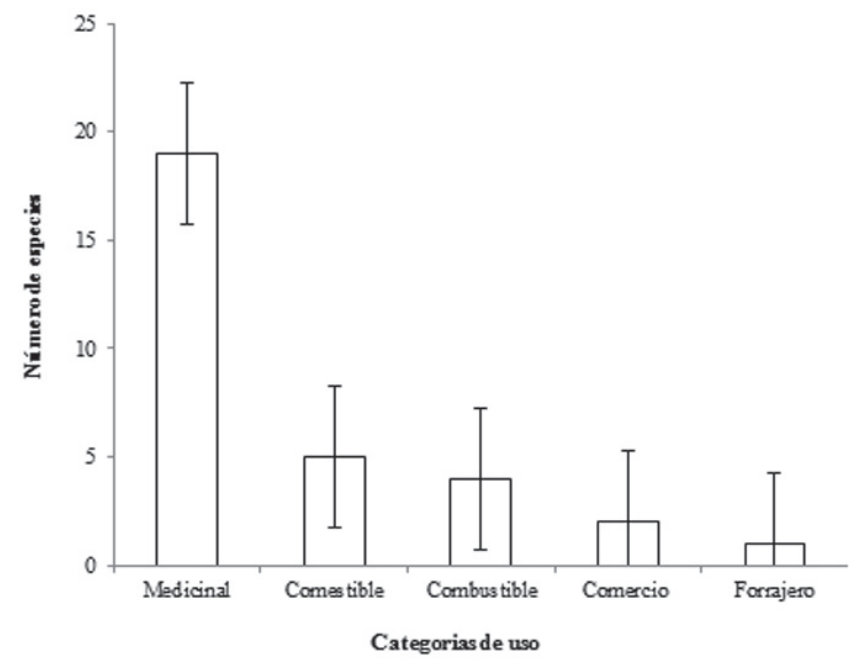

Figura 2. Categorías de uso con respecto al número de plantas mencionadas. La barra representa el error típico estándar. 


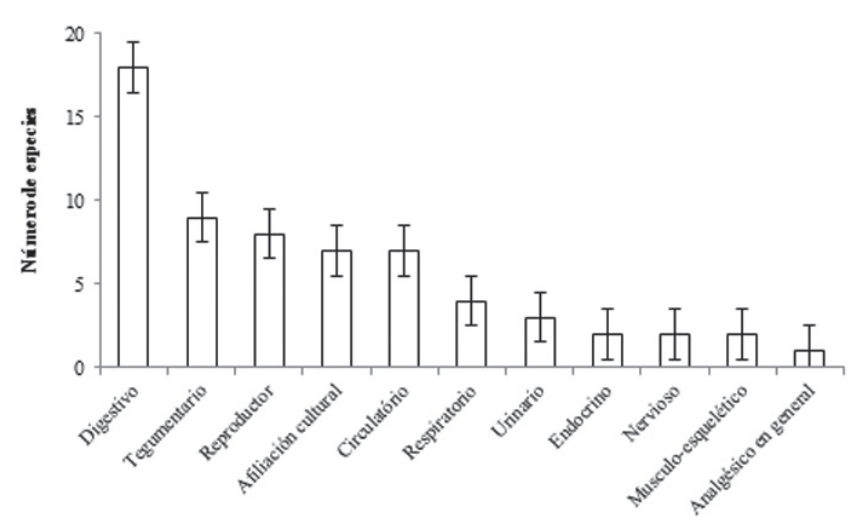

Figura 3. Aparatos y sistemas tratados por número de plantas medicinales. La barra representa el error típico estándar.

ocho padecimientos: dolor de próstata, regular la menstruación, cólicos menstruales, infección en la matriz, dolor de ovarios, facilitar el parto, infecciones vaginales y aborto. Los padecimientos mencionados que no se reconocen por la medicina alopática, y sin embargo están presentes en la concepción de enfermedad de los informantes de la comunidad, fueron siete, y se agruparon en la categoría de afiliación cultural, siendo baños para parturientas, aire en el estómago, aire de muerto, chincual, mal aire, espanto o susto y mal de ojo. El sistema tegumentario presentó seis padecimientos: heridas, granos, inflamación por golpes, caída de cabello, infecciones en la piel y fuegos labiales. El aparato circulatorio agrupó cuatro padecimientos: regular la presión arterial, varices, mejorar la circulación y afecciones en el corazón; el respiratorio tres: tos, asma y dolor de garganta. El sistema urinario tres padecimientos: afecciones en riñón, mal de orín y cálculos renales; sistema endócrino únicamente con diabetes; sistema nervioso con insomnio y nervios alterados; sistema músculo-esquelético con dolor muscular o de huesos y como analgésico únicamente dolor de cabeza.

La proporción de acuerdo entre informantes respecto a las plantas medicinales utilizadas para tratar determinados aparatos o sistemas, presentó mayor homogeneidad en Costus scaber, Plantago major, Psidium guajava, Jaegeria macrocephala y Equisetum myriochaetum (Cuadro 2). Costus scaber es utilizada para padecimientos de riñón y mal de orín; P. major en gastritis, dolor de estómago y dolor de riñón; P. guajava para dolor de estómago, diarrea, disentería, colitis, indigestión y vómito y; J. macrocephala para gastritis, lavar heridas, dolor de estómago, ulceras, golpes y granos en la piel. Los resultados obtenidos mostraron que el sistema urinario (0.77) tratado con Bidens pilosa, C. scaber, E. myriochaetum y P. major; afiliación cultural (0.43) tratados principalmente con Justicia spicigera y Sambucus nigra canadensis; así como el aparato digestivo (0.41) trata-

Cuadro 2. Estructuras usadas (EU): $\mathrm{H}=$ hoja, $\mathrm{FI}=$ flor, $\mathrm{Fr}=$ fruto, $\mathrm{T}=$ tallo. Proporción de acuerdo entre informantes sobres aparatos y sistemas tratados por las plantas medicinales. El resultado se registró entre 0 y 1 , en donde el valor más alto indica mayor acuerdo y entre más se aleje de 1, es menor. Dig: digestivo, Res: respiratorio, Uri: urinario, Rep: reproductor, ACu: afiliación cultural, Te: tegumentario, MEs: músculo-esquelético, Cir: circulatorio, Ner: nervioso, End: endócrino, Ana: analgésico en general y PA: proporción total de acuerdo para las especies.

\begin{tabular}{|c|c|c|c|c|c|c|c|c|c|c|c|c|c|}
\hline Especies & EU & Dig & Res & Uri & Rep & $\mathrm{ACu}$ & $\mathrm{Te}$ & MEs & Cir & Ner & End & Ana & PA \\
\hline Bidens pilosa & $\mathrm{H}, \mathrm{Fl}$ & 0.18 & & 0.82 & & & & & 0.12 & & 0.24 & & 0.34 \\
\hline Cecropia obtusifolia & $\mathrm{H}$ & & & & & & & 0.11 & 0.22 & & 0.56 & & 0.30 \\
\hline Citrus limetta & $\mathrm{H}, \mathrm{Fl}, \mathrm{Fr}$ & 0.40 & & & & & & & 0.20 & 0.13 & & 0.07 & 0.20 \\
\hline Citrus $\times$ aurantium & $\mathrm{H}, \mathrm{Fl}, \mathrm{Fr}$ & & 0.29 & & & & & & & 0.35 & & 0.18 & 0.26 \\
\hline Costus scaber & $\mathrm{H}, \mathrm{T}$ & & & 1.00 & & & & & & & & & 1.00 \\
\hline Cymbopogon citratus & $\mathrm{H}$ & 0.80 & & & & & & & & 0.20 & & & 0.50 \\
\hline Equisetum myriochaetum & $\mathrm{H}, \mathrm{T}$ & 0.23 & & 0.91 & & & & & & & & & 0.57 \\
\hline Guazuma ulmifolia & $\mathrm{H}, \mathrm{Fr}$ & 0.67 & & & & 0.17 & & & & & 0.25 & & 0.36 \\
\hline Hamelia patens & $\mathrm{H}$ & 0.60 & 0.44 & & 0.12 & & 0.44 & 0.04 & & & 0.04 & 0.04 & 0.24 \\
\hline Jaegeria macrocephala & $\mathrm{H}, \mathrm{FI}$ & 0.50 & & & & & 0.94 & & & & & & 0.72 \\
\hline Justicia spicigera & $\mathrm{H}, \mathrm{Fl}$ & 0.05 & & & & 0.64 & 0.14 & & 0.32 & & & 0.05 & 0.21 \\
\hline Kalanchoe pinnata & $\mathrm{H}$ & 0.09 & & & & 0.18 & 0.36 & & & & & & 0.23 \\
\hline Passiflora edulis & $\mathrm{H}, \mathrm{Fr}$ & 0.22 & & 0.11 & & & & & 0.11 & & & & 0.14 \\
\hline Persea americana & $\mathrm{H}, \mathrm{Fr}$ & 0.24 & & & 0.41 & & & & 0.12 & & & & 0.25 \\
\hline Phyla dulcis & $\mathrm{H}$ & 0.56 & 0.06 & & 0.06 & 0.38 & 0.06 & & 0.06 & & & & 0.18 \\
\hline Plantago major & $\mathrm{H}$ & 0.09 & & 1.00 & & & & & & & & & 0.54 \\
\hline Pseudogynoxys chenopodioides & $\mathrm{H}, \mathrm{FI}$ & 0.69 & & & & & 0.81 & 0.06 & & & & & 0.41 \\
\hline Psidium guajava & $\mathrm{H}, \mathrm{Fr}$ & 1.00 & & & & & & & & & & & 1.00 \\
\hline \multirow[t]{2}{*}{ Sambucus nigra subsp. canadensis } & $\mathrm{H}, \mathrm{Fl}$ & 0.24 & 0.24 & & & 0.81 & 0.10 & & & & & & 0.30 \\
\hline & & 0.41 & 0.26 & 0.77 & 0.16 & 0.43 & 0.41 & 0.07 & 0.16 & 0.23 & 0.23 & 0.08 & \\
\hline
\end{tabular}


Cuadro 3. Prácticas de manejo registradas de las plantas medicinales en la comunidad de Cantarranas. TL: tolerada, PT: protegida, PM: promovida, RS: recolección selectiva. Origen. i: introducida, n: nativa, in: incierto. Los superíndices de las especies hacen referencia al número de registro bajo el que se encuentran en el herbario (HGOM).

\begin{tabular}{|c|c|c|c|c|c|c|c|c|c|c|}
\hline \multirow[b]{3}{*}{ Especies } & \multirow{3}{*}{ Origen } & \multirow{3}{*}{ Recolección } & \multicolumn{7}{|c|}{ Prácticas de manejo } & \multirow{3}{*}{ Cultivo } \\
\hline & & & \multicolumn{3}{|c|}{$\begin{array}{c}\text { Manejo no } \\
\text { selectivo incipiente }\end{array}$} & \multicolumn{4}{|c|}{$\begin{array}{c}\text { Manejo selectivo } \\
\text { incipiente }\end{array}$} & \\
\hline & & & $\mathrm{TL}$ & PT & PM & RS & $\mathrm{TL}$ & PT & PM & \\
\hline Bidens pilosa mavn1597 & $\mathrm{n}$ & $x$ & $x$ & $x$ & $x$ & & & & & \\
\hline Cecropia obtusifolia mavn1576 & $\mathrm{n}$ & $x$ & & & & & & & & \\
\hline Citrus limetta vzh131 & $\mathrm{i}$ & $x$ & $x$ & $\mathrm{x}$ & $x$ & & $\mathrm{x}$ & $x$ & $x$ & \\
\hline Citrus $\times$ aurantium vzh84 & $\mathrm{i}$ & & $\mathrm{x}$ & $x$ & $x$ & $\mathrm{x}$ & $\mathrm{x}$ & $x$ & $x$ & \\
\hline Costus scaber mavn922 & $\mathrm{n}$ & $x$ & & & & & $x$ & $x$ & $x$ & $x$ \\
\hline Cymbopogon citratus mavn573 & $\mathrm{i}$ & & & & & & & & $x$ & $\mathrm{x}$ \\
\hline Equisetum myriochaetum mavn1831 & $\mathrm{n}$ & $x$ & & & & & & & & \\
\hline Guazuma ulmifolia bnlg1 & $\mathrm{n}$ & $x$ & $x$ & $\mathrm{x}$ & $x$ & & & & & \\
\hline Hamelia patens mavn1687 & $\mathrm{n}$ & $x$ & $\mathrm{x}$ & $\mathrm{x}$ & $x$ & $x$ & $\mathrm{x}$ & $x$ & $x$ & \\
\hline Jaegeria macrocephala mavn1556 & $\mathrm{n}$ & $x$ & $\mathrm{x}$ & $x$ & $\mathrm{x}$ & $\mathrm{x}$ & $x$ & $x$ & $x$ & \\
\hline Justicia spicigera mavn1656 & $\mathrm{n}$ & & & & & & $x$ & $x$ & $x$ & \\
\hline Kalanchoe pinnata ${ }^{\text {mavn875 }}$ & $\mathrm{i}$ & $x$ & & & & & & & & \\
\hline Pasiflora edulis mavn1654 & $\mathrm{i}$ & & & & & & $x$ & & $x$ & $x$ \\
\hline Persea americana mavn1674 & $\mathrm{n}$ & $x$ & & & & & $x$ & $\mathrm{x}$ & & $\mathrm{x}$ \\
\hline Phyla dulcis ytc157 & $\mathrm{n}$ & $x$ & $\mathrm{x}$ & $\mathrm{x}$ & $x$ & & & & $x$ & \\
\hline Plantago major mavn888 & $\mathrm{i}$ & & $\mathrm{x}$ & $\mathrm{x}$ & $\mathrm{x}$ & & & & & \\
\hline Pseudogynoxys chenopodioides ytc140 & $\mathrm{n}$ & $x$ & $x$ & $\mathrm{x}$ & $x$ & & $x$ & $x$ & $x$ & \\
\hline Psidium guajava mavn1730 & in & & & & & & & $\mathrm{x}$ & $x$ & $x$ \\
\hline Sambucus nigra subsp. canadensis mavn1073 & $\mathrm{n}$ & $x$ & $x$ & $x$ & $x$ & & & & $x$ & \\
\hline
\end{tabular}

do con Cymbopogon citratus, P. guajava y Pseudogynoxys chenopodioides, presentaron los valores más elevados en la proporción de acuerdo. También se observa que Hamelia patens es la especie que se utiliza para tratar el mayor número de aparatos o sistemas (7; Cuadro 2).
Se mencionó que el $33 \%$ de las plantas medicinales se obtienen de la huerta, $18 \%$ de caminos, $16 \%$ de los patios o jardines cercanos a las casas, $10 \%$ de las milpas y del monte, $7 \%$ cerca del río y $6 \%$ de potreros. La "huerta", es referida por los habitantes como una propiedad alejada de la casa en

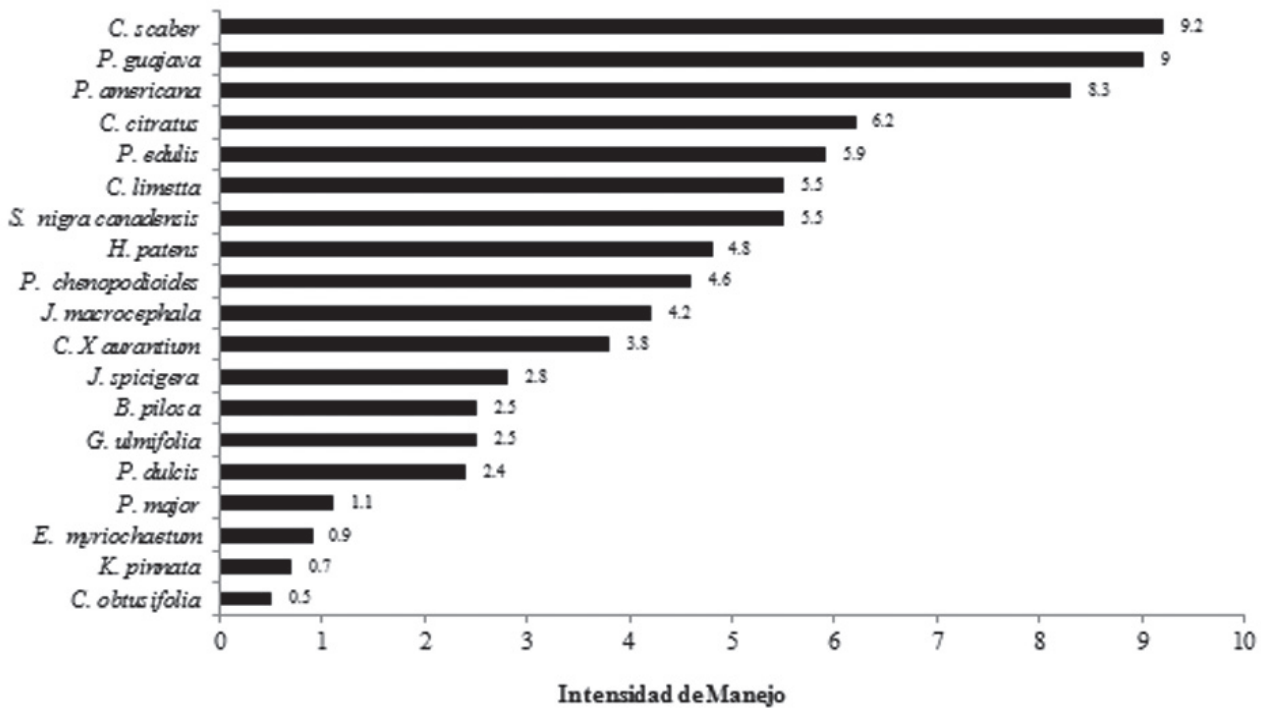

Figura 4. Intensidad de manejo de las plantas medicinales en la comunidad de Cantarranas. 


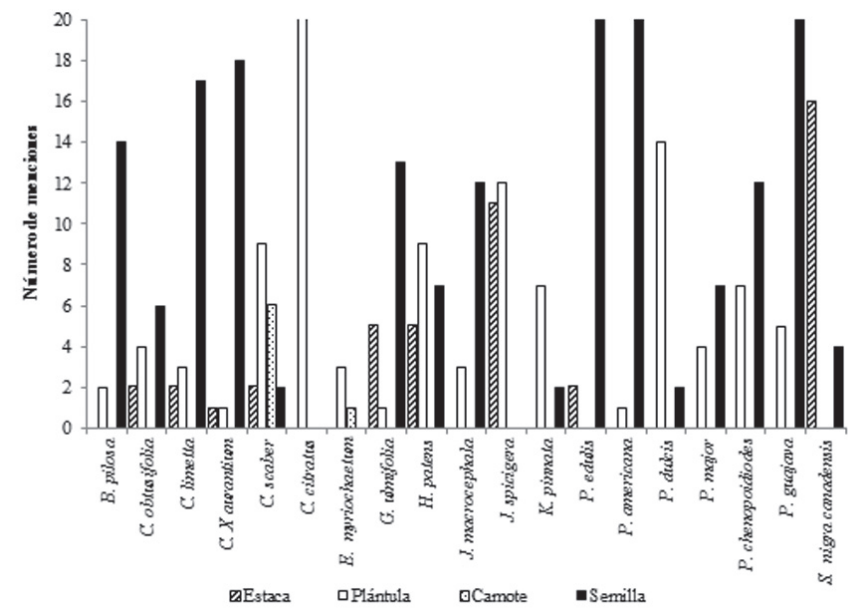

Figura 5. Propagación tradicional de plantas medicinales en Cantarranas. Información derivada de las entrevistas aplicadas a informantes clave.

donde principalmente se cultiva café y crecen plantas de la flora nativa sometidas a cierto grado de manejo. Las plantas medicinales se encuentran sometidas a diferentes prácticas de manejo (Cuadro 3). La mayoría de éstas (68\%) se obtienen mediante recolección simple en poblaciones silvestres y además presentan manejo selectivo, no selectivo y/o cultivo. Las especies que presentaron manejo más especializado fueron Costus scaber, Cymbopogon citratus, Passiflora edulis, Persea americana y Psidium guajava bajo cultivo.
No se observó una práctica preferente en el manejo no selectivo; sin embargo, en el manejo selectivo la promoción y protección de las plantas son las actividades más comunes.

La intensidad de manejo de las plantas medicinales osciló entre 0.5 a 9.2 (Figura 4). Los valores más altos de intensidad de manejo corresponden a plantas bajo cultivo y con demanda en el mercado, derivado de su uso medicinal y comestible; por ejemplo, Costus scaber, Persea americana y Psidium guajava. Los valores más bajos, se presentan en Cecropia obtusifolia, Equisetum myriochaetum y $\mathrm{Ka}$ lanchoe pinnata, manejadas únicamente bajo recolección simple en poblaciones silvestres. La intensidad de manejo se encuentra relacionada de manera positiva con respecto al valor cultural de las especies ( $\mathrm{R}=0.67, P=0.002)$; es decir, las especies con mayor valor cultural presentan mayor intensidad de manejo; esta tendencia se obtuvo sin considerar a E. myriochaetum, que presenta características notablemente distintas al resto. La forma de propagación tradicional más común es por medio de plántula, en 16 de las 19 especies; y por semilla en 15 especies; $C$. scaber presentó las cuatro vías de propagación registradas (estaca, plántula, camote y semilla), a diferencia de Cymbopogon citratus que únicamente se propaga por plántulas (Figura 5). Las formas de propagación se reportan como fueron mencionadas por los informantes; la plántula hace referencia al trasplante de plantas individuales completas; estaca y camote a la propagación utilizando partes vegetativas.

Durante los recorridos en la zona de estudio se locali-
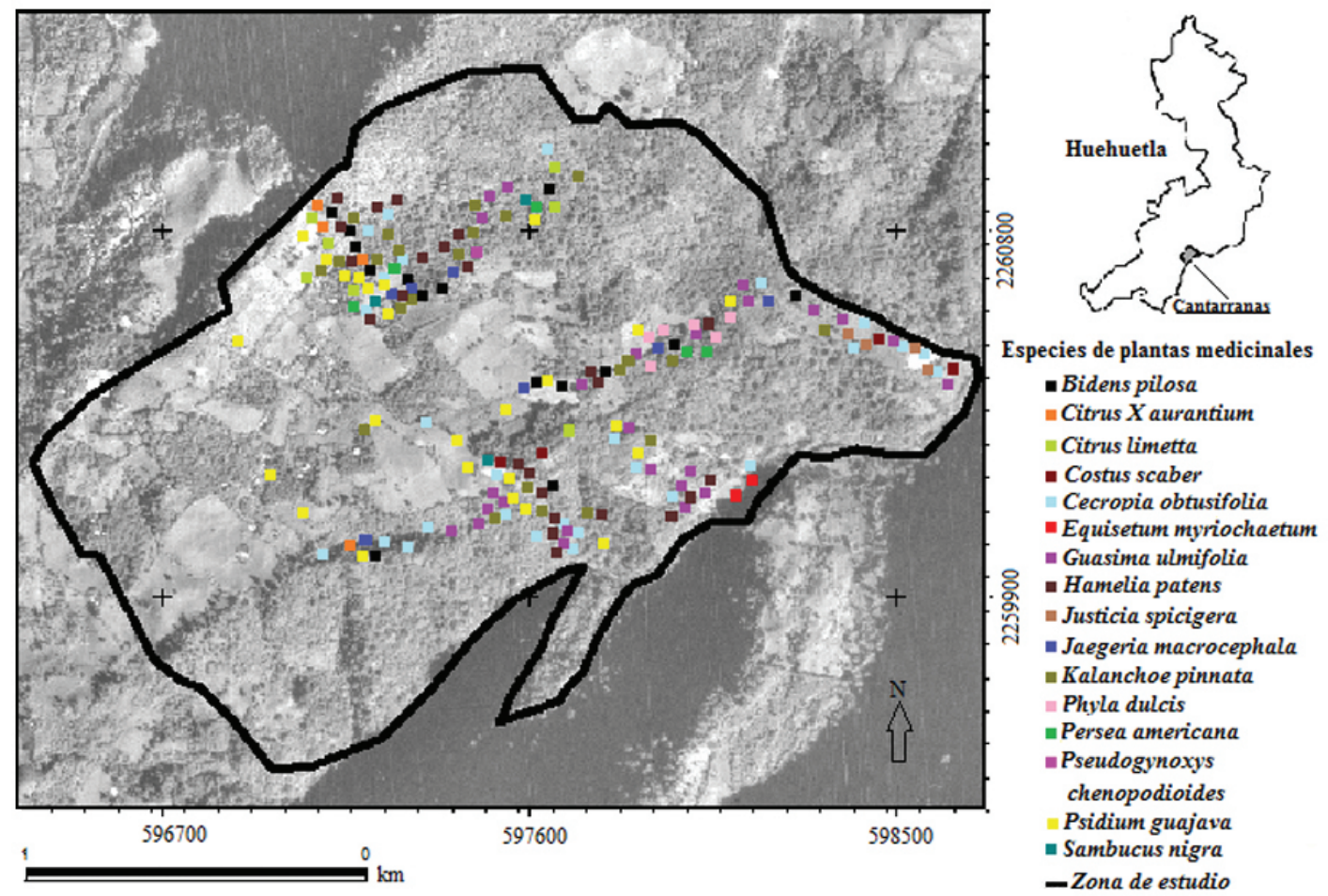

Figura 6. Distribución geográfica de las poblaciones de plantas medicinales localizadas en los recorridos de campo en la comunidad de Cantarranas. 


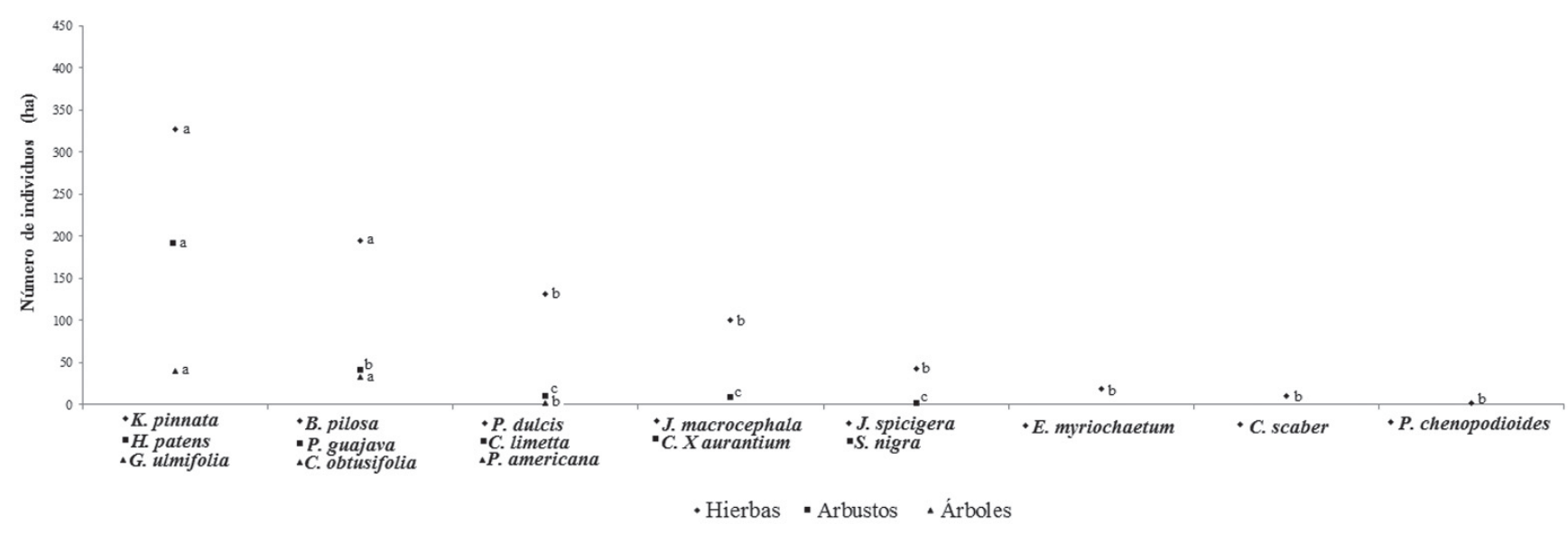

Figura 7. Promedio de la densidad poblacional por hectárea de las 16 plantas medicinales localizadas en la zona de estudio. Se presentan por separado las tres formas de vida consideradas para cada una de las especies. Las letras representan las diferencias estadísticas entre la densidad poblacional.

zaron 16 de las 19 especies consideradas en este trabajo, en altitudes de 390 a 1,260 m s.n.m. (Figura 6). El 56\% de las 16 especies localizadas (Citrus $\times$ aurantium, Costus scaber, Citrus limetta, Equisetum myriochaetum, Guazuma ulmifolia, Justicia spicigera, Persea americana, Pseudogynoxys chenopodioides y Sambucus nigra subsp. canadensis) presentaron en promedio cobertura rara, $13 \%$ cobertura ocasional (Cecropia obtusifolia y Psidium guajava) y frecuente (Jaegeria macrocephala y Phyla dulcis) y, 18\% abundante (Bidens pilosa, Hamelia patens y Kalanchoe pinnata). Las especies que no se localizaron en los recorridos fueron Plantago major, Cymbopogon citratus y Pasiflora edulis, derivado de que son especies introducidas y sin poblaciones silvestres; pero son cultivadas en patios o jardines y sometidas a uso y manejo al igual que el resto de las especies.

La densidad poblacional de las especies localizadas oscila entre 1 y 387 individuos por hectárea, según la especie y la forma de vida (Figura 7). Las especies hierbas: Bidens pilosa y Kalanchoe pinnata difirieron significativamente en cuanto a su densidad poblacional de Costus scaber, Equisetum myriochaetum, Jaegeria macrocephala, Justicia spicigera, Phyla dulcis y Pseudogynoxys chenopodioides $(\mathrm{H}=$ 32.11, $P=0.0008$ ). Los arbustos Hamelia patens y Psidium guajava difieren entre ellos y de Citrus limetta, Citrus $\times$ aurantium y Sambucus nigra subsp. canadensis $(\mathrm{H}=21.6, P$ $=0.0006$ ); y los tres árboles registrados Guazuma ulmifolia y Cecropia obtusifolia difieren significativamente de Persea americana $(\mathrm{H}=10.8, P=0.005)$. Las especies que difieren por su baja densidad poblacional son consideradas más vulnerables a ser afectadas por un manejo no sostenible; por lo que su uso y aprovechamiento debe considerar su disponibilidad en la zona de estudio. La densidad poblacional no presentó relación con la intensidad de manejo ni con el valor cultural; sin embargo, conocer datos ecológicos de las plantas medicinales es un punto de partida para planificar su conservación. La densidad poblacional presentó una correlación significativa con respecto a la disponibilidad que los informantes perciben de éstas $(\mathrm{R}=0.54, P=0.01)$. En el caso de $B$. pilosa y $K$. pinnata, los informantes tienen claro que son las especies más abundantes en la zona de estudio, y por el contrario E. myriochaetum y J. macrocephala son percibidas como raras, tal como se registró en los recorridos de campo. Lo anterior, corrobora la importancia de documentar el conocimiento tradicional que tienen los pobladores acerca del manejo de las plantas medicinales y su participación en proyectos de conservación.

Cuadro 4. Remedios y medicamento herbolario elaborados por la Sociedad Cooperativa Flor de Corpus de Cantarranas S.C. R.L.

\begin{tabular}{|c|c|c|c|c|c|}
\hline Nombre científico & Nombre común & Presentación & Parte utilizada & Uso & $\begin{array}{l}\text { Vía de } \\
\text { administración }\end{array}$ \\
\hline $\begin{array}{l}\text { Equisetum myriochaetum } \\
\text { y Costus scaber }\end{array}$ & $\begin{array}{l}\text { cola de caballo } \\
\text { y caña de jabalí }\end{array}$ & remedio herbolario & hojas, tallo & sistema urinario & oral \\
\hline $\begin{array}{l}\text { Psidium guajava, Hamelia } \\
\text { patens y Plantago major }\end{array}$ & $\begin{array}{l}\text { guayaba, tres hojitas } \\
\text { y llantén }\end{array}$ & remedio herbolario & hojas & aparato digestivo & oral \\
\hline $\begin{array}{l}\text { Jaegeria macrocephala, } \\
\text { Pseudogynoxys chenopodioides } \\
\text { y Hamelia patens }\end{array}$ & $\begin{array}{l}\text { árnica, árnica de } \\
\text { bejuco y tres hojitas }\end{array}$ & $\begin{array}{l}\text { medicamento } \\
\text { herbolario (pomada) }\end{array}$ & hojas, flor & sistema tegumentario & externa \\
\hline
\end{tabular}




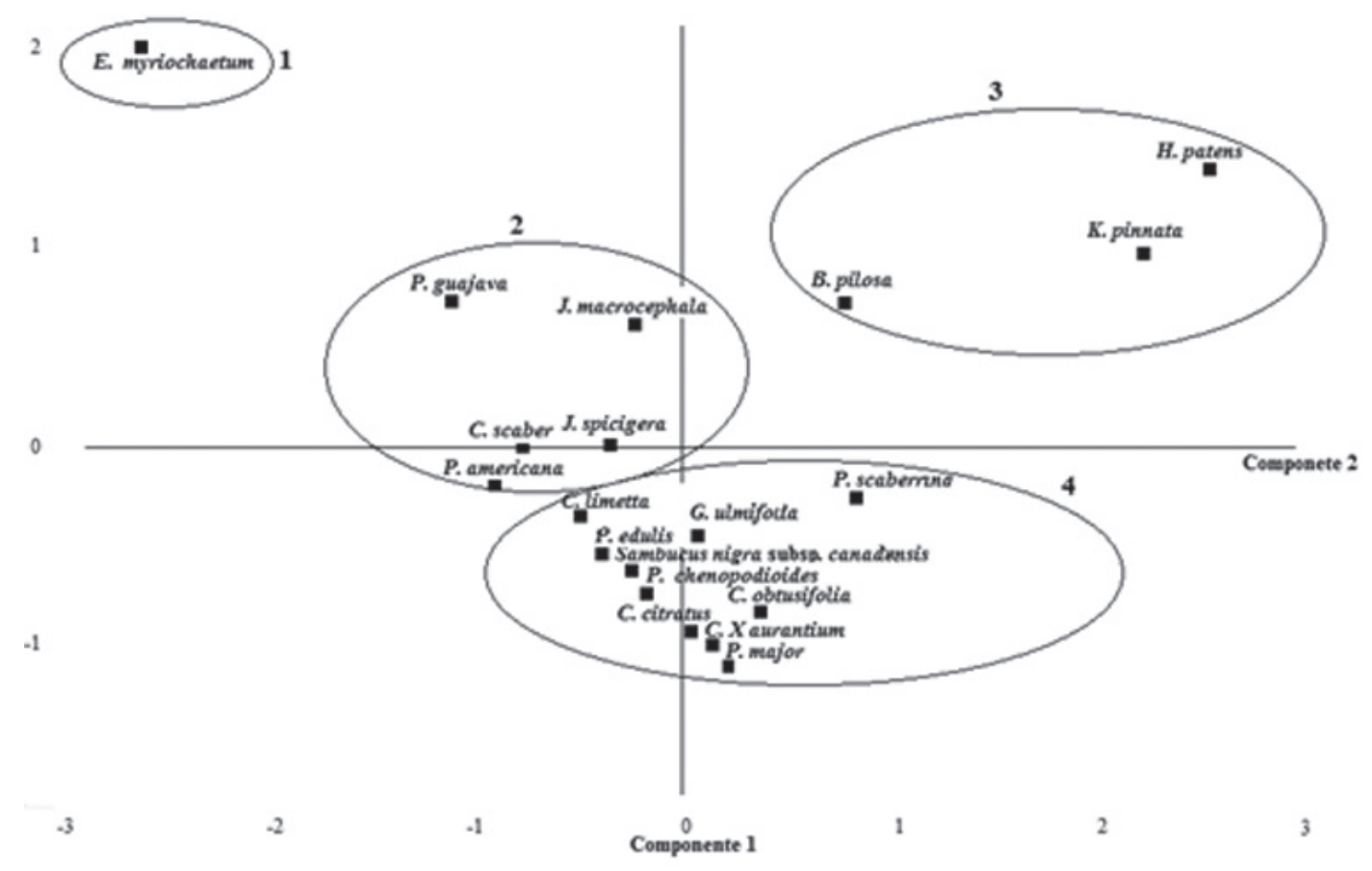

Figura 8. Proyección de las especies en el espacio dada por las variables que proporcionaron más peso como resultado del Análisis de Componentes Principales. Los dos primeros componentes explican $76.8 \%$ de la variación.

El análisis de componentes principales explica $76.8 \%$ de la variación de los datos por los dos primeros componentes (Figura 8), lo que permite la distribución de las especies en cuatro grupos. El grupo 1 y 2 definidos por los valores más altos en la variable de valor cultural, el grupo 3 por su mayor densidad poblacional, el grupo 4 por valores intermedios tanto de importancia cultural, densidad poblacional e índice de manejo.

Se constituyó legalmente un grupo bajo el régimen de Sociedad Cooperativa, con el nombre de "Flor de Corpus de Cantarranas S.C. de R.L.", conformada por siete mujeres de la comunidad. Trabajan actualmente con el objetivo de aprovechar de manera sostenible y conservar las plantas medicinales y sus hábitats en la comunidad. A los miembros de dicha organización y pobladores en general se les impartió un diplomado (100 horas) llamado "Uso y aprovechamiento sostenible de plantas medicinales del estado de Hidalgo"; certificado por la Dirección de Vinculación y Educación Continua de la Universidad Autónoma del Estado de Hidalgo. En dicho diplomado se impartieron cinco cursos y cuatro talleres con temas como: la importancia de la diversidad cultural y biológica de México e Hidalgo, uso de las plantas medicinales importantes de la zona de estudio, recolecta sostenible, técnicas de cultivo, construcción de huertos, procesamiento de plantas medicinales y elaboración de remedios herbolarios. Considerando el valor cultural, nivel de fidelidad, acuerdo entre informantes, antecedentes bibliográficos, experiencias satisfactorias del uso de plantas y los padecimientos registrados en la comuni- dad, las miembros de la Sociedad Cooperativa definieron y elaboraron remedios herbolarios (Cuadro 4); los cuales son distribuidos y utilizados como opción para mejorar la salud dentro de la comunidad, incrementar el ingreso económico de las familias involucradas y evitar el aprovechamiento no sostenible de las plantas involucradas.

\section{Discusión}

Conocer datos sobre el uso, importancia cultural, manejo tradicional, comercio, distribución geográfica e importancia ecológica de las plantas medicinales son herramientas para llevar a cabo su aprovechamiento sostenible y conservación (OMS et al., 1993; Hersch-Martínez, 1995,1996; CBD, 2002; Hamilton, 2004; Singh et al., 2012; Bhat et al., 2013; Blancas et al. 2013). Trabajos realizados en diferentes países del mundo (Adams et al., 2004; Hamilton, 2004, 2008; Lange, 2004; Wiersum et al., 2006; van de Kop et al., 2006; Shengji et al., 2010; Raina et al., 2011; Rajeswara et al., 2012) han destacado que el aprovechamiento sostenible de plantas medicinales debe ser considerado una estrategia para conservar la biodiversidad, mejorar la calidad de vida y mitigar la pobreza en comunidades que dependan de éstas; teniendo como respaldo el significado que las plantas medicinales representan para la población en materia de salud, ambiente, economía e identidad cultural.

Equisetum myriochaetum, Psidium guajava, Jaegeria macrocephala, Costus scaber, Persea americana, Justicia spicigera y Bidens pilosa son consideradas las plantas 
medicinales con mayor valor cultural en la comunidad de Cantarranas. Investigaciones previas en torno a la flora medicinal de la zona Otomí-Tepehua realizadas en San Bartolo Tutotepec y Huehuetla, mencionan entre los primeros lugares de importancia cultural (valor de uso) las mismas especies reportadas en este trabajo (Callejas et al., 2008; Zavala-Fragoso, 2011). Lo anterior, destaca la importancia de dichas plantas a nivel regional y la necesidad de la elaboración de estrategias sobre su uso, ya que aparentemente, el aprovechamiento de las plantas medicinales en la región no se realiza bajo ningún esquema sostenible.

Se registraron 51 padecimientos tratados por las 19 plantas estudiadas, el $84 \%$ de éstas son utilizadas para tratar el aparato digestivo. Se utilizan principalmente especies como: Psidium guajava, Cymbopogon citratus, Pseudogynoxys chenopodioides, Guazuma ulmifolia, Hamelia patens y Phyla dulcis. Esta tendencia se ha observado en países como Italia, Perú, Tanzania, Bolivia y México (Amri y Kisangau, 2012; Quiroga et al., 2012; Monigatti et al., 2013; Vitalini et al., 2013; Loredo-Medina et al. 2002; MolinaMendoza et al., 2012); por tanto, es importante conocer, conservar y disponer de las especies usadas para ese fin, en tanto que las enfermedades gastrointestinales causadas por bacterias, virus o parásitos representan 2.2 millones de muertes cada año a nivel mundial (OMS, 2011).

Las prácticas de manejo de las plantas medicinales, presentaron un gradiente de manipulación a través de actividades relacionadas con el aumento de su disponibilidad. El gradiente se presenta de menor (recolección simple en poblaciones silvestres) a mayor especialización (cultivo) y se relaciona directamente con la intensidad de manejo. Lo anterior se ha reportado en el manejo de plantas comestibles y ha sugerido que la intensidad de manejo es una respuesta asociada a la seguridad alimentaria, y que se relaciona principalmente con la importancia económica y valor cultural, facilidad de propagación de las especies y disponibilidad (González-Insuasti y Caballero, 2006; Blancas et al., 2013). $\mathrm{La}$ intensidad de manejo de las plantas medicinales en Cantarranas se relaciona directamente con su valor cultural, a excepción de Equisetum myriochaetum, que es la especie con mayor importancia cultural pero presentó intensidad de manejo bajo, atribuida a su difícil propagación según la experiencia de los informantes. Costus scaber, Cymbopogon citratus, Persea americana, Passiflora edulis y Psidium guajava presentaron las intensidades de manejo más altas y son especies manipuladas mediante el cultivo. Costus scaber es utilizada únicamente como medicinal, de lo cual deriva su intensidad de manejo y destaca su importancia en la comunidad; a diferencia de $C$. citratus, $P$. americana, $P$. edulis y $P$. guajava que presentan valor comestible y comercial, a lo que se atribuye principalmente su alta intensidad de manejo.

El 74\% de las plantas medicinales incluidas en este trabajo se recolectan de poblaciones silvestres. Lo anterior presenta una tendencia mundial con respecto al aprove- chamiento (Wang, 1999; Shengji et al., 2010; Amri y Kisangau, 2012; Blancas et al., 2013), por lo que los hábitats silvestres son considerados reservorios para la mayoría de las plantas (Amri y Kisangau, 2012). Muchas de las plantas medicinales provienen de hábitats perturbados (Stepp, 2004); Cantarranas presenta sitios con estas características (huertas, milpas, potreros y caminos) de los cuales los pobladores obtienen plantas medicinales y comestibles. Las reservas extractivas (Pérez-García y Rebollar, 2004) y áreas de conservación comunitaria (Camacho et al., 2010) son hábitats naturales o modificados que contienen valores significativos de biodiversidad, funciones ecológicas, valores culturales y que son conservados voluntariamente por las comunidades locales. Lo anterior, constituye una estrategia para la protección de las plantas medicinales y las áreas en donde se realiza su extracción, evitando su pérdida continua y acelerada que afecta a la biodiversidad. En Cantarranas se detectaron sitios con características significativas para su conservación. El primer sitio, se argumenta por la presencia de Equisetum myriochaetum, debido a que es una especie con elevado valor cultural a nivel local y regional, es recolectada de poblaciones silvestres para uso medicinal y venta en mercados de la zona, presenta restringida distribución geográfica y muy baja densidad poblacional. Por lo anterior, se considera necesaria la protección de su hábitat, en el que se localizan especies como Protium copal, Cojoba arborea y Platanus mexicana, mencionadas en la lista roja de árboles mexicanos de bosque nublado (The Red List of Mexican Cloud Forest Trees) bajo la categoría NT (Near Threatened; González-Espinosa et al., 2011). El segundo sitio propuesto para su protección es un fragmento localizado de bosque de Quercus sp.; argumentando que dichos bosques en zonas de clima caliente de México constituyen condiciones redictuales en donde el clima era más fresco y se relaciona con glaciaciones del pleistoceno (Sousa, 1968; Pennington y Sarukhán, 1998). La erosión de estos bosques provoca desecación de manantiales, contaminación del agua e inundaciones, por lo que es urgente su conservación en donde su presencia es necesaria para preservar al equilibrio ecológico y las especies que ahí se localizan (Rzedowski, 1978).

Si bien, la recolecta de plantas medicinales en poblaciones silvestres es una actividad que se ha desarrollado durante mucho tiempo y bajo prácticas de manejo tradicional; existen factores que ponen en riesgo la disponibilidad de las plantas, tales como la pérdida de hábitat, cambio climático y extracción no sostenible. Por lo anterior, se consideró como una estrategia de conservación la recolecta sostenible (OMS, 2003; MPSG, 2006; OMS et al., 1993) aplicada para las plantas medicinales con amplia distribución geográfica y densidad poblacional elevada, tales como: Bidens pilosa, Cecropia obtusifolia, Guazuma ulmifolia, Hamelia patens, Kalanchoe pinnata y Psidium guajava. El cultivo asegura la disponibilidad y reduce la extracción en hábitats silvestres de las plantas medicinales, por lo que es considerado 
una herramienta para la conservación de la biodiversidad (Wiersum et al., 2006; Hamilton, 2008; Raina et al., 2011; Rajeswara et al., 2012). Se propuso el cultivo de Equisetum myriochaetum, Costus scaber, Jaegeria macrocephala, Pseudogynoxys chenopodioides, Justicia spicigera, Sambucus nigra y Persea americana, ya que son especies con elevada importancia cultural, pero presentan restringida distribución geográfica y baja densidad poblacional. Con lo anterior, se pretende evitar que dichas especies sigan siendo extraídas de poblaciones silvestres. Derivado del trabajo de la Sociedad Cooperativa conformada en Cantarranas, se han establecido tres huertos de plantas medicinales, considerados como un reservorio fitogenético de especies nativas e introducidas (Gispert et al., 1993, 2010). En los huertos, actualmente se cultivan de manera tradicional y experimental las 19 plantas estudiadas; algunas de ellas completaron sus ciclo de vida y las miembros de la Sociedad Cooperativa, las aprovechan de manera sostenible para elaborar remedios herbolarios (Ley General de Salud, 1984). Los remedios herbolarios son distribuidos y utilizados como opción para mejorar la salud de los pobladores y así mismo incrementar el ingreso económico de las familias involucradas, repercutiendo en la mejora de la calidad de vida en la comunidad.

El análisis de componentes principales es útil para distinguir cuatro grupos con respecto al valor cultural y densidad poblacional de las plantas medicinales. El primer grupo, lo conforma únicamente Equisetum myriochaetum, que es la especie con mayor importancia cultural y alto nivel de fidelidad en el tratamiento de afecciones del aparato urinario. Equisetum myriochaetum es comúnmente comercializado y manejado mediante la recolecta en poblaciones silvestres; por lo que presentó baja intensidad de manejo, distribución geográfica restringida y baja densidad poblacional. El grupo dos se conforma por especies con alto valor cultural y con alta probabilidad de ser efectivas, por lo cual las especies de este grupo se consideran confiables para uso medicinal; además de ser medicinales presentan valor comestible, son sometidas a prácticas de manejo selectivo incipiente y/o cultivo, lo que les confiere altas intensidades de manejo. En el grupo tres se encuentran especies con amplia distribución geográfica en la zona de estudio y alta densidad poblacional, sometidas a prácticas de manejo simples (recolección en poblaciones silvestres y manejo no selectivo). El grupo cuatro se conforma por especies que tienen valores intermedios de importancia cultural, intensidad de manejo, frecuencia de uso y densidad poblacional. Al considerar los resultados del análisis de componentes principales, características de uso y manejo, valor cultural, distribución y densidad poblacional, se definieron, con ayuda de las miembros de la Sociedad Cooperativa, algunas especies prioritarias para la comunidad en matera de uso, aprovechamiento y conservación.

Equisetum myriochaetum es usada para afecciones en el aparato urinario, las cuales se mencionan entre las diez principales causas de enfermedad del estado de Hidalgo
(Secretaria de Salud, 2009). Es una especie con alta demanda en el mercado, considerada bajo el estatus biológico de recolectada (no cultivada) y nativa (originaria de México) (Martínez-Moreno et al., 2006). El género tienen importancia medicinal, farmacológica, fitoquímica, cultural, histórica y comercial en comunidades del interior de la República Mexicana, es usada para infecciones renales, dolores corporales, enfermedades del aparato digestivo como dolor de estómago, gastritis y úlceras, cálculos biliares, mal de orín, afecciones del hígado y problemas relacionados con la menstruación (Martínez, 1985; Argueta, 1994; Gallardo-Pérez et al., 2006). Se ha encontrado que no presenta toxicidad con referencia a signos clínicos, peso corporal, análisis de orina, hematología bioquímica ni lesiones histopatológicas (Tago et al., 2010). Los fitoextractos de E. myriochaetum sustentan su uso en la medicina tradicional, presentando ausencia de genotoxicidad y actividad antimutágena, lo que valida así su uso para el tratamiento de pacientes con diabetes en forma de té (Castañeda et al., 2011).

Costus scaber presenta alto nivel de fidelidad en el tratamiento de afecciones en el sistema urinario. Es una especie originaria de México, utilizada en la India como ornamental, en donde la gente local come las hojas y la raíz como antihelmíntico; su propagación es muy rápida en suelo, por medio de raíces y vástagos; los extractos de sus hojas presentan actividad hipoglucemiante, por lo que es importante la promoción de su cultivo, para así hacer crecer el desarrollo y disponibilidad de una medicina natural para pacientes diabéticos (Benny, 2004). De igual manera se han demostrado sus efectos antidiabéticos con extractos de hojas, presentando reducción de glucosa y lípidos en sangre, incremento en niveles de insulina plasmática, proteínas y glucógeno hepático, sin efectos tóxicos en riñón, hígado y páncreas (Jothivel et al., 2007). Si bien, en Cantarranas, $C$. scaber es utilizada para tratar afecciones renales, debe considerarse que este es uno de los principales padecimientos ocasionados por la diabetes mellitus (Nathan, 1996).

Jaegeria macrocephala, Hamelia patens y Psidium guajava son especies consideradas con alto valor cultural a nivel regional, son utilizadas principalmente para tratar afecciones de la piel y aparato digestivo, al igual que lo reportado por Zavala-Fragoso (2011) y Callejas et al. (2008), quienes mencionan que estas especies ocupan los primeros sitios de importancia cultural de la flora medicinal en Huehuetla y San Bartolo Tutotepec. Psidium guajava presenta actividad antibacteriana significativa con extractos metanólicos de las hojas y se ha definido como segura en uso terapéutico (Anas et al., 2008; Ismail et al., 2012).

Tras la evidente problemática presente a nivel mundial en términos de pérdida de biodiversidad, en diferentes países se han promovido proyectos con iniciativas de aprovechamiento sostenible y conservación basados en plantas medicinales; con el objetivo de mejorar la salud, dar sustento a la población rural, garantizar el suministro futuro de plantas 
medicinales y el cultivo como una herramienta para la conservación de la biodiversidad (Lange, 2004; Wiersum et al., 2006; Hamilton, 2008; Shengji et al., 2010). Este trabajo se presentó como un modelo para el aprovechamiento de plantas medicinales, de forma que se respete la integridad funcional y la capacidad de carga de los ecosistemas de los cuales forman parte (Ley General de Equilibrio Ecológico y la Protección al Ambiente, 1988) mediante actividades de investigación, organización y educación comunal. Además, se implementó la relación de la Institución de Educación Superior con organizaciones de la sociedad civil, por medio de la vinculación (Campos y Sánchez, 2005, 2006). La cual promueve el desarrollo de servicios tecnológicos, asesorías, capacitación, educación continua, inserción de estudiantes y docentes a los sectores privados y sociales, y el aprovechamiento de los recursos humanos formados en las Instituciones, para impulsar al desarrollo social y humano de los grupos con mayor vulnerabilidad.

\section{Agradecimientos}

El presente estudio contó con el apoyo económico del proyecto FOMIX-CONACYT HGO-2010-C01-151064 "Plantas medicinales y hongos de Hidalgo: aprovechamiento sustentable y conservación como estrategias para mejorar la calidad de vida". Se extiende un agradecimiento a los revisores del artículo. A los informantes y a la comunidad en general de Cantarranas, Huehuetla, Hidalgo por su colaboración en el trabajo; especialmente a la señora Victoria Solís.

\section{Literatura citada}

Adams W.M., Aveling R., Brockington D., Dickson B., Elliott J., Hutton J., Roe D., Vira B. y Wolmer W. 2004. Biodiversity conservation and the eradication of poverty. Science 306:11461149.

Alcántara A.O. y Luna V.I. 1997. Florística y análisis biogeográfico del bosque mesófilo de montaña de Tenango de Doria, Hidalgo, México. Anales del Instituto de Biología, Universidad Nacional Autónoma de México, Serie Botánica 68:57-106.

Alexiades M.N. 1996. Selected Guidelines for Ethnobotanical Research: A Field Manual. New York Botanical Garden, Nueva York.

Amri E. y Kisangau D.P. 2012. Ethnomedicinal study of plants used in villages around Kimboza forest reserve in Morogoro, Tanzania. Ethnobiology and Ethnomedicine 8:1.

Anas K., Jayasree P.R., Vijayakumar T. y Kumar P.R.M. 2008. In vitro antibacterial activity of Psidium guajava Linn. leaf extract on clinical isolates of multidrug resistant Staphylococcus aureus. Indian Journal of Experimental Biology 46:41-46.

Argueta A.1994. Atlas de las Plantas de la Medicina Tradicional Mexicana I, II, III. Instituto Nacional Indigenista, México, D.F.

Benny M. 2004. Insulin plant in gardens. Natural Product Radiance 3:349-350.

Bermúdez A., Oliveira-Miranda M.A. y Velázquez D. 2005. La in- vestigación etnobotánica sobre plantas medicinales. Una revisión de sus objetivos y enfoques actuales. Interciencia 30:453-459.

Bhat J.A., Kumar M. y Bussmann R.W. 2013. Ecological status and traditional knowledge of medicinal plants in Kedarnath Wildlife Sanctuary of Garhwal Himalaya, India. Journal of Ethnobiology and Ethnomedicine 9:1.

Blancas J., Casas A., Pérez-Salicrup D., Caballero J. y Vega E. 2013. Ecological and socio-cultural factors influencing plant management in Náhuatl communities of the Tehuacán Valley, México. Journal of Ethnobiology and Ethnomedicine 9:39.

Boster J.S. 1985. Requiem for the omniscient informant: there's life in the old girl yet. En: Dougherty J.W.D. Ed. Directions in Cognitive Anthropology, pp. 177-197, University of Illinois Press, Urbana.

CMMAD. Comisión Mundial sobre el Media Ambiente y el Desarrollo. 1987. Nuestro futuro común. Organización de las Naciones Unidas. <http://www.un.org/es/comun/docs/?symbol= A/42/427> (consultado 24 de junio, 2013).

Bye R. 1998. La intervención del hombre en la diversificación de las plantas en México. En: Ramamoorthy T.P., Bye R., Lot A. y Fa J. Eds. Diversidad Biológica de México, pp. 689-713. Instituto de Biología, Universidad Nacional Autónoma de México, México, D.F.

Caballero J. y Cortés L. 2001. Percepción, uso y manejo tradicional de los recursos vegetales en México. En: Rendón A.B., Rebollar D.S., Caballero N.J. y Martínez A.M.A. Eds. Plantas Cultura y Sociedad, pp. 79-100, Universidad Autónoma Metropolitana, Secretaria del Medio Ambiente, Recursos Naturales y Pesca, México, D.F.

Callejas C.M., Pérez E.B.E. y Villavicencio N.M.A. 2008. Flora Medicinal de San Bartolo Tutotepec, Hidalgo. Universidad Autónoma del Estado de Hidalgo, Pachuca.

Camacho B.C., Martín G.J. y del Campo-García C. 2010. Fortalecimiento de áreas de conservación comunitaria e indígenas a través de la etnobiología. En: Moreno F.A., Pulido S.M.T., Mariaca M.R., Valadez A.R., Mejía C.P. y Gutiérrez S.T.V. Eds. Sistemas Biocognitivos Tradicionales: Paradigmas en la Conservación Biológica y el Fortalecimiento Cultural, pp. 30-35, Asociación Etnobiológica Mexicana, A. C., Global Diversity Foundation, Universidad Autónoma del Estado de Hidalgo; El Colegio de la Frontera Sur y Sociedad Latinoamericana de Etnobiología, México, D.F.

Campos R.G. y Sánchez D.G. 2005. La vinculación Universitaria: Ese oscuro objeto del deseo. Revista Electrónica de Investigación Educativa 7(2) <http://redie.uabc.mx/index.php/redie/ article/view/134/230> (consultado 16 de febrero, 2013).

Campos R.G. y Sánchez D.G. 2006. La vinculación universitaria y sus interpretaciones. Ingenierías 30:18-25.

Castañeda S.A.N., Ordaz T.M.G., Andrade-Cetto A., Segal K.C. y Rodríguez-Arnaiz R. 2011. Antimutagenic activity of two medicinal phytoextracts in somatic cells of Drosophila melanogaster. Pharmaceutical Biology 49:640-647.

CBD. Conservation of Biological Diversity. 2002. Estrategia Global para la Conservación Vegetal. Organización de las Naciones Unidas. <https://www.cbd.int/doc/publications/pc-brochure -es. pdf $>$ (consultado 15 de junio, 2013).

COESPO. Consejo Estatal de Población. 2010. Hidalgo: Población total, indicadores socioeconómicos, índice y grado de marginación y lugar que ocupa en los contextos nacional y estatal por localidad, 2010. <http://poblacion.hidalgo.gob.mx/ 
descargables /Hgo_localidades_marginacion2010.pdf > (consultado 25 junio, 2013).

de Schutter A. 1981. Investigación Participativa: Una Opción Metodológica para la Educación de Adultos. Centro Regional para la Educación de Adultos y Alfabetización Funcional para América Latina (CREFAL), Pátzcuaro.

Durston J. y Miranda F. 2002. Experiencias y metodología de la investigación participativa. Organización de las Naciones Unidas. <http://www.eclac.org/publicaciones/xml/4/10204/ lc11715-p.pdf $>$ (consultado 8 de noviembre, 2013).

ESRI. Environmental Systems Research Institute. 1999. Using Arc View@ database access (Version 2.1a). Environmental Systems Research Institute, Inc., Nueva York.

Friedman J., Yaniv Z., Dafni A. y Palewitch D. 1986. A preliminary classification of the healing potential of medicinal plants, based on a rational analysis of an ethnopharmacological field survey among Bedouins in the Negev Desert, Israel. Journal Ethnopharmacology 16:275-287.

Gallardo-Pérez J.C., Esparza-Aguilar M.L. y Gómez-Campos A. 2006. Importancia etnobotánica de las plantas vasculares sin semilla en México: Equisetum. Polibotánica 21:61-74.

Gispert C.M., Diego P.N., Jiménez R.J., Gómez A., Quintanilla J. y García L. 1979. Un nuevo enfoque en la metodología etnobotánica en México. Medicina Tradicional 2:41-52.

Gispert C.M., Gómez A. y Núñez P.A. 1993. Concepto y manejo tradicional de los huertos familiares en dos bosques tropicales mexicanos. En: Leff E. y Carabias J. Eds. Cultura y Manejo Sustentable de los Recursos Naturales, pp. 576-623, Miguel Ángel Porrúa, México, D.F.

Gispert C.M., Vales G.M.A. y Vilamajó A.D. 2010. Els horts familiars de Mèxic i Cuba Interrelació existent entre l'entorn natural, la societat i les identitats culturals a l'Amèrica tropical. Revista d'Etnologia de Catalunya 36:104-114.

Gómez A.R. 2012. Plantas medicinales en una aldea del estado de Tabasco, México. Revista Fitotecnia Mexicana 35:43-49.

González-Chévez L. y Hersch-Martínez P. 1993. Aportes para la construcción del perfil epidemiológico sociocultural de una comunidad rural. Salud Publica de México 35:393-402.

González-Insuasti M.S. y Caballero J. 2006. Managing plant resources: how intensive can it be? Human Ecology 35:303314.

González-Espinosa M., Meave J.A., Lorea-Hernández F.G., Ibarra-Manríquez G. y Newton A.C. 2011. The Red List of Mexican Cloud Forest Trees. Fauna \& Flora international, Cambridge.

Goodman L. 1961. Snowball sampling. Annals of Mathematical Statistics 32:148-170.

Guzmán G.F. 2009. Diagnóstico sobre la Situación de Mujeres y Hombres en el Municipio de Huehuetla, Hidalgo. Instituto Hidalguense de las Mujeres, Pachuca.

Hamilton A.C. 2004. Medicinal plants, conservation and livelihoods. Biodiversity and Conservation 13:1477-1517.

Hamilton A.C. 2008. Medicinal Plants in Conservation and Development: Case Studies and Lessons Learnt. Plantlife International, Salisbury.

Heinrich M., Ankli A., Frei B., Weimann C. y Sticher O. 1998. Medicinal plants in México: healers' consensus and cultural importance. Social Science and Medicine 47:1859-1871.

Hersch-Martínez P. 1995. Commercialization of wild medicinal plants from southwest Puebla, Mexico. Economic Botany 49:197-206.
Hersch-Martínez P. 1996. Destino Común. Los Recolectores y su Flora Medicinal. Instituto Nacional de Antropología e Historia, México, D.F.

Hersch-Martínez P. y González C.L.1996. Investigación participativa en etnobotánica. Algunos procedimientos coadyuvantes en ella. Dimensión Antropológica 8:129-153.

INEGI. Instituto Nacional de Estadística y Geografía. 1992. Síntesis geográfica del estado de Hidalgo. Instituto Nacional de Estadística, Geografía e Informática, Aguascalientes.

INEGI. Instituto Nacional de Estadística y Geografía. 2000. <http:// www.inegi.org.mx/sistemas/olap/Proyectos/bd/censos/cpv2000/ PTL.asp?s=est\&c=10261\&proy=cpv00_pt?s=est\&c=10261> (consultado 5 marzo, 2013).

INEGI. Instituto Nacional de Estadística y Geografía. 2004. La Población Hablante de Lengua Indígena de Hidalgo. <http:// www.inegi.org.mx/prod_serv/contenidos/espanol/bvinegi/productos/censos/poblacion/poblacion_indigena/PerLi_Hgo.pdf> (consultado 13 de noviembre, 2013).

Ismail M., Minhas P.S., Khanum F., Sahana V.M. y Sowmya C. 2012. Antibacterial activity of leaves extract of Guava (Psidium Guajava). International Journal of Research in Pharmaceutical and Biomedical Sciences 3:1-2.

Jothivel N., Ponnusamy S.P., Appachi M., Singaravel S., Rasilingam D., Deivasigamani K. y Thangavel S. 2007. Anti-diabetic activity of metanol leaf extract of Costus scaber D. Don in alloxan-induced diabetic rats. Journal of Health Science 53:655-663.

Krebs C.J. 1999. Ecological Methodology. University of British Columbia, Nueva York.

Kumar M., Sheikh M.A. y Bussmann R. 2011. Ethnomedicinal and ecological status of plants in Garhwal Himalaya, India. Journal of Ethnobiology and Ethnomedicine 7:32.

Ley General de Equilibrio Ecológico y la Protección al Ambiente. 1988. <http://www.diputados.gob.mx/LeyesBiblio/pdf/148. pdf $>$ (consultado 8 de noviembre, 2013).

Ley General de Salud. 1984. <http://www.stps.gob.mx/bp/ secciones/dgsst/normatividad/142.pdf> (consultado 10 septiembre, 2013)

Linares E. y Bye R. 2009. La dinámica de un mercado periférico de plantas medicinales de México: el tianguis de Ozumba, Estado de México, como centro acopiador para el mercado de Sonora (mercado central). En: Long T.J. y Attolini L.A Eds. Caminos y Mercados de México, pp. 631-663, Instituto de Investigaciones Históricas, Universidad Nacional Autónoma de México, Instituto Nacional de Antropología e Historia, México, D.F.

Linares M.E., Bye R. y Flores P.B. 1990. Tés Curativos de México. Instituto de Biología, Universidad Nacional Autónoma de México, México, D.F.

Lagos-Witte S., Sanabria D.O.L., Chacón P. y García R. 2011. Manual de Herramientas Etnobotánicas Relativas a la Conservación y el Uso Sostenible de los Recursos Vegetales. Red Latinoamericana de Botánica, Santiago de Chile.

Lange D. 2004. Medicinal and aromatic plants: trade, production, and management of botanical resources. Acta Horticulturae 629:177-197.

Loredo-Medina O.L., Rodríguez-Chávez J.M. y Ramos-Espinosa M.G. 2002. Aprovechamiento de recursos vegetales en una localidad de la Reserva de la Biosfera Mariposa Monarca, Michoacán, México. Etnobiología 2:32-60.

Martínez M. 1985. Catálogo de Nombres Vulgares y Científicos de 
Plantas Mexicanas. Fondo de Cultura Económica, México, D.F.

Martínez A.M.A. 1990. Investigaciones etnobotánicas en la Sierra Norte de Puebla. En: Leff E., Carabias J. y Batis A.I. Eds. Recursos Naturales, Técnica y Cultura. Estudios y Experiencias para un Desarrollo Alternativo, pp. 55-74, Centro de Investigaciones Interdisciplinario en Humanidades, Universidad Nacional Autónoma de México, México, D.F.

Martínez-Moreno D., Alvarado-Flores R., Mendoza-Cruz M. y Basurto-Peña F. 2006. Plantas medicinales de cuatro mercados del estado de Puebla, México. Boletín de la Sociedad Botánica de México 79:79-87.

Mendoza C.G. y Lugo P.R. 2011. Plantas Medicinales en los Mercados de México. Universidad Autónoma Chapingo, Chapingo.

Mendoza C.G., García P.J. y Estrada L.E. 1997. Catálogo y usos terapéuticos de plantas medicinales que se comercializan en fresco en el mercado de Sonora. Materiales para la docencia No. 2, Universidad Autónoma Chapingo, Chapingo.

Molina-Mendoza J.L., Galván-Villanueva R., Patiño-Siciliano A. y Fernández-Nava R. 2012. Plantas medicinales y listado florístico preliminar del municipio de Huasca de Ocampo, Hidalgo, México. Polibotánica 34:239-271.

Monigatti M., Bussmann R.W. y Weckerle C.S. 2013. Medicinal plant use in two Andean communities located at different altitudes in the Bolívar Province, Peru. Journal of Ethnopharmacology 145:450-464.

Monroy R. y Ayala I. 2003. Importancia del conocimiento etnobotánico frente al proceso de urbanización. Etnobiología 3:79-92.

Mostacedo B. y Fredericksen T.S. 2000. Manual de Métodos Básicos de Muestreo y Análisis en Ecología Vegetal. Proyecto de Manejo Forestal Sostenible, Santa Cruz.

MPSG. Medicinal Plant Specialist Group. 2006. Estándar Internacional para la Recolección Silvestre Sostenible de Plantas Medicinales y Aromáticas (ISSC-MAP). <http://www.floraweb. de/map-pro/Standard_Version1_0_spanisch.pdf> (consultado 29 de noviembre, 2013).

Nathan D.M. 1996. The pathophysiology of diabetic complications: How much does the glucose hypothesis explain? Annals of Internal Medicine 124: 86-89.

Nicholson M.S. y Arzeni C.B. 1993. The market medicinal plants of Monterrey, Nuevo León, México. Economic Botany 47:184192.

OMS. Organización Mundial de la Salud. 2003. Directrices de la OMS sobre buenas prácticas agrícolas y de recolección (BPAR) de plantas medicinales. <http://apps.who.int/medicinedocs/pdf/ s5527s/s5527s.pdf $>$ (consultado 8 de octubre, 2013).

OMS. Organización Mundial de la Salud. 2011. Estadísticas sanitarias mundiales. <http://www.who.int/whosis/whostat/ES_ WHS2011_Full.pdf> (consultada 10 de septiembre, 2013).

OMS. Organización Mundial de la Salud,UICN. Unión Mundial para la Naturaleza y WWF. Fondo Mundial para la Naturaleza. 1993. Directrices sobre conservación de plantas medicinales. <http://www.urosario.edu.co/urosario_files/57/571bf298-6ad84b7f-b432-26a6fb78e6de.pdf> (consultada 8 de noviembre, 2013).

Pavón N.P. y Meza S.M. 2009. Cambio Climático en el Estado de Hidalgo: Clasificación y Tendencias Climáticas. Universidad Autónoma del Estado de Hidalgo, Pachuca.

Payyappallimana U. y Fadeeva Z. 2013. Innovation in Local and Global Learning Systems for Sustainability. Traditional Knowledge and Biodiversity-Learning Ccontributions of the
Regional Centres of Expertise on Education for sustainable Development. United Nations University, Institute of Advanced Studies, Yokohama.

Pennington D.T. y Sarukhán J. 1998. Árboles Tropicales de México. Manual para la Identificación de las Principales Especies. $2^{\mathrm{a}}$ ed. Universidad Nacional Autónoma de México, Fondo de Cultura Económica, México, D.F.

Pérez E.B.E., Villavicencio N.M.A. y Ramírez A.A. 2003. Lista de las Plantas Útiles del Estado de Hidalgo. Universidad Autónoma del Estado de Hidalgo, Pachuca.

Pérez-García M. y Rebollar D.S. 2004. Reservas extractivas ¿Alternativa para la conservación de especies forestales? Madera y Bosques 10:55-69.

Pieroni A. 2001. Evaluation of the cultural significance of wild food botanicals traditionally consumed in northwestern Tuscany, Italy. Journal of Ethnobiology 21:89-104.

Quiroga R., Meneses L. y Bussmann R.W. 2012. Medicinal ethnobotany in Huacareta (Chuquisaca, Bolivia). Journal of Ethnobiology and Ethnomedicine 8:29.

Raina R., Chand R. y Sharma Y.P. 2011. Conservation strategies of some important medicinal plants. International Journal of Medicinal and Aromatic Plants 1:342-347.

Rajeswara R.B.R., Syamasundar K.V., Rajput D.K., Nagaraju G. y Adinarayana G. 2012. Biodiversity, conservation and cultivation of medicinal plants. Journal of Pharmacognosy 3(2):59-62.

Rodríguez N.J. y Torres B. 1990. Importancia de la conservación de la biodiversidad biológica y cultural para el manejo integrado de los recursos. En: Leff E., Carabias J y Batis A.I. Eds. Recursos Naturales, Técnica y Cultura. Estudios y Experiencias para un Desarrollo Alternativo, pp. 23-40, Centro de Investigaciones Interdisciplinario en Humanidades, Universidad Nacional Autónoma de México, México, D.F.

Romero L.E., Santiago A.A. y Basilio G.C. 1999. Plantas Medicinales y de Otros Usos de San Antonio el Grande, Huehuetla, Hidalgo, en Yuhu (Otomí de la Sierra). Universidad Autónoma del Estado de Hidalgo, Pachuca.

Rubin de Celis T.E. 1988. Investigación científica vs investigación participativa: reflexiones en torno a una falsa disyuntiva. Reflexiones en torno a una falsa disyuntiva. En: Vío G.F., Gianotten V. y de Wit T. Eds. Investigación Participativa y Praxis Rural.Nuevos Conceptos de Educación y Desarrollo Local, pp. 123-137, Consejo de Educación de Adultos de América Latina, Santiago de Chile.

Rzedowski J. 1978. Vegetación de México. Limusa, México, D.F.

Sánchez J.M.L y Estrada L.E. 1995. Distribución de 10 plantas medicinales mexicanas. Su medio ecológico y cultural (Zapote blanco, Guarumbo, Chaya mansa, Mala mujer, Guácima, Muicle, Berro, Zarzaparrilla, Tronadora y Chichicastle). En: Estrada L.E. Eds. Lecturas para el Diplomado Internacional Plantas Medicinales de México, pp. 579, Universidad Autónoma Chapingo, Chapingo.

SAS Institute. 2007. JMP user guide, release 7. SAS Institute Inc., Cary.

Schäfer C. 2001. Traditional knowledge relating to the conservation and sustainable use of biodiversity. <http://www. conservation-development.net/Projekte/Nachhaltigkeit/CD1/ LaenderDesSuedens/Themenblaetter/PDF/Biodiv_TK_engl_ traditional_knowledge.pdf $>$ (consultado 10 de julio, 2013).

Secretaria de Salud. 2009. Carpeta de Información Estratégica. Estado de Hidalgo. Secretaría de Salud, México, D.F. 
SEMARNAT. Secretaria de Medio Ambiente y Recursos Naturales. 2010. Norma Oficial Mexicana NOM-059-SEMARNAT2010. <http://dof.gob.mx/nota_detalle.php?codigo=5173091 $\&$ fecha $=30 / 12 / 2010>$ (consultado 6 de mayo, 2013).

Shengji P., Hamilton A.C., Lixin Y., Huyin H., Zhiwei Y., Fu G. y Quangxin Z. 2010. Conservation and development through medicinal plants: a case study from Ludian (Northwest Yunnan, China) and presentation of a general model. Biodiversity and Conservation 19:2619-2636.

Singh H., Husain T., Agnihotri P., Pande P.C. e Iqbal M. 2012. Biodiversity conservation through traditional beliefs system: a case study from Kumaon Himalayas, India. International Journal of Conservation Science 3:33-40.

Sousa M. 1968. Ecología de las leguminosas de Los Tuxtlas, Veracruz. Anales del Instituto de Biología, Universidad Nacional Autónoma de México, Serie Botánica 1:121-160.

Stepp J.R. 2004. The role of weeds as sources of pharmaceuticals. Journal of Ethnopharmacology 92:163-166.

Sutherland W.J. 1996. Ecological Census Techniques: A Handbook. Cambridge University Press, Nueva York.

Tago Y., Wei M., Ishii N., Kakehashi A. y Wanibuchi H. 2010. Evaluation of the subchronic toxicity of dietary administered Equisetum arvense in F344 rats. Journal Toxicology Pathology 23:245-251.

van de Kop P., Alam G. y Piters B.S. 2006. Developing a sustainable medicinal-plant chain in India. En: Ruben, R., Slingerland M. and Nijhoff H. Eds. Agro-Food Chains and Networks for Development, pp. 191-202, Springer, Dordrecht.

Villavicencio N.M.A y Pérez E.B.E. 2005. Guía de la Flora Útil de la Huasteca y la Zona Otomí-Tepehua, Hidalgo I. Universidad Autónoma del Estado de Hidalgo, Pachuca.

Villavicencio N.M.A. y Pérez E.B.E. 2006. Plantas Útiles del Estado de Hidalgo III. Universidad Autónoma del Estado de Hidalgo, Pachuca.

Vitalini S., Iriti M., Puricelli C., Ciuchi D. y Segale A. y Fico G. 2013. Tradicional knowledge on medicinal and food plants used in Val San Giocomo (Sondrio, Italy)-An alpine ethnobotanical study. Journal of Ethnopharmacology 145:517-529.

Wang Y.H. 1999. Sustainable management of medicinal plant resource in Northwest of Yunnan: The case of Ludian administrative Village in Lijiang County. Tesis doctoral, Kunming Institute of Botany, Chinese Academy of Sciences, Kunming. 295 pp.

Wiersum K., Dold A.P., Husselman M. y Cocks M. 2006. Cultivation of medicinal plants as a tool for biodiversity conservation and poverty alleviation in the Amatola region, South Africa. En: Bogers R.J., Craker L.E. y Lange D. Eds. Medicinal and Aromatic Plants: Agricultural, commercial, ecological, legal, pharmacological, and social aspects, pp. 43-57, Springer, Berlín.

WHO. World Health Organization. 2002. Traditional medicine strategy 2002-2005. World Health Organization, Ginebra.

Zar J.H. 1996. Biostatistical Analysis. 3a ed. Prentice-Hall, Englewood Cliffs.

Zavala-Fragoso A. 2011. Plantas medicinales de la cabecera municipal de Huehuetla, Hidalgo. Tesis de licenciatura, Centro de Investigaciones Biológicas, Universidad Autónoma del Estado de Hidalgo, Pachuca, 239 pp.

Recibido: 23 de julio de 2013

Aceptado: 23 de noviembre de 2013 